nephron

Practice

\title{
UK Renal Registry 17th Annual Report: Chapter 3 Demographic and Biochemistry Profile of Kidney Transplant Recipients in the UK in 2013: National and Centre-specific Analyses
}

\author{
Rishi Pruthi ${ }^{a}$, Anna Casula ${ }^{a}$, lain MacPhee ${ }^{b}$

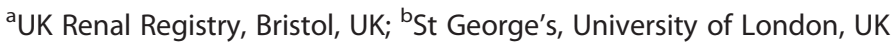

\section{Key Words}

Blood pressure - Bone metabolism - Chronic kidney disease . Clinical Commissioning Group - Deceased donor - eGFR . Epidemiology · Ethnicity · Graft function - Haemoglobin · Live donor - Outcomes · Renal transplantation - Survival

\footnotetext{
Abstract

Introduction: National transplant registries routinely focus on centre-specific patient and graft survival rates following renal transplantation. However other outcomes such as graft function (as measured by eGFR), haemoglobin and blood pressure are also important quality of care indicators. Methods: Renal transplant activity, incident graft survival data and donor information were obtained from NHS Blood and Transplant. Laboratory and clinical variables and prevalent survival data were obtained from the UK Renal Registry. Data were analysed separately for prevalent and one year post-transplant patients. Results: The main
}

increase in transplant activity in 2013 was the use of donors after brainstem death. The death-censored graft failure rate was similar to previous years at $2.4 \%$ and the transplant patient death rates remained stable at 2.4 per 100 patient years. There was centre variation in outcomes including eGFR and haemoglobin in prevalent and 1 year posttransplant patients. Analysis of prevalent transplants by chronic kidney disease stage showed $13.4 \%$ with an eGFR $<30 \mathrm{ml} / \mathrm{min} / 1.73 \mathrm{~m}^{2}$ and $1.7 \%$ with an eGFR $<15 \mathrm{ml} / \mathrm{min} /$ $1.73 \mathrm{~m}^{2}$. Of those with CKD stage 5T, 32.4\% had haemoglobin concentrations $<100 \mathrm{~g} / \mathrm{L} 28.4 \%$ phosphate concentrations $\geqslant 1.7 \mathrm{mmol} / \mathrm{L}$ and $16.8 \%$ adjusted calcium concentrations $\geqslant 2.5 \mathrm{mmol} / \mathrm{L}$. Infection (26\%) and malignancy (24\%) remained amongst the commonest causes of death in patients with a functioning renal transplant. Conclusion: Significant variations in clinical outcomes (unadjusted for patient specific variables) amongst kidney transplant recipients continued to exist in the UK and may reflect differences in healthcare delivery between renal centres. 


\section{Introduction}

This chapter includes independent analyses regarding renal transplant activity and survival data from the UK Transplant Registry, held by the Organ Donation and Transplantation Directorate (ODT) of NHS Blood and Transplant (NHSBT). The UK Renal Registry (UKRR) has performed additional analyses of renal transplant recipient follow-up data examining demographics, clinical and biochemical variables. NHSBT records all the information regarding the episode of transplantation (donor and recipient details) and the UKRR holds additional information on key clinical and biochemical variables in renal transplant recipients. The co-operation between these two organisations results in a comprehensive database describing the clinical care delivered to renal transplant patients within the UK. This further allows for the comparison of key outcomes between centres and provides insight into the processes involved in the care of such patients in the UK.

This chapter is divided into six sections: (1) transplant activity, waiting list and survival data; (2) transplant demographics; (3) clinical and laboratory outcomes; (4) analysis of prevalent patients by chronic kidney disease (CKD) stage; (5) eGFR slope analysis; and (6) cause of death in transplant recipients. Methodology, results and conclusions of these analyses are discussed in detail for all six sections separately.

The UK Renal Registry methodology is described elsewhere [1]. The UKRR collects quarterly clinical data via an electronic data extraction process from hospital based renal IT systems on all patients receiving renal replacement therapy. Throughout the chapter, the number preceding the centre name in each figure indicates the percentage of missing data for that centre for that variable.

Unless otherwise specified, prevalent transplant patients were defined as patients with a functioning renal transplant on the 31st December 2013.

A list of the recommended audit measures from the Renal Association which are relevant to the transplant population are given in appendix 1 of this chapter. Several of the audit measures are not currently reported by the UKRR in the annual report; the reasons behind this are varied, but predominantly relate to a high proportion of incomplete data or that the relevant variable is not currently within the specified UKRR dataset. Over time it is hoped to work with the renal community to improve reporting across the range of recommended standards.

\section{Transplant activity, waiting list activity and survival data}

\section{Introduction}

NHSBT prospectively collects donor and recipient data around the episode of transplantation. They also request that transplant centres provide an annual paper based data return on the status of the recipient's graft function. This enables ODT to generate comprehensive analyses of renal transplant activity and graft survival statistics.

NHSBT attributes a patient to the centre that performed the transplant operation irrespective of where the patient was cared for before or after the procedure and hence only reports on transplant centre performance.

\section{Methods}

In 2013, there were 23 UK adult renal transplant centres, 19 in England, two in Scotland and one each in Northern Ireland and Wales.

Comprehensive information from 1999 onwards concerning the number of patients on the transplant waiting list, the number of transplants performed, the number of deceased kidney donors (donor after brainstem death and donor after circulatory death), living kidney donors, patient survival and graft survival is available on the NHSBT website (http://www.organdonation.nhs.uk/ukt/ statistics/statistics.asp).

\section{Results}

During 2013, 3,257 kidney or kidney plus other organ transplants were performed. The absolute number of living kidney donors showed a 6\% rise in 2013 representing $33.8 \%$ of all transplants performed whilst donor after circulatory death transplants continued to increase and comprised $24.4 \%$ of all kidney transplants performed. A $20 \%$ rise in the number of transplants from donors after brainstem death was also noted in 2013 (table 3.1).

There were small differences in one and five year risk-adjusted patient and graft survival rates amongst UK renal transplant centres (table 3.2). These graft survival rates include grafts with primary non-function (which are excluded from analysis by some countries).

Using data from the UKRR on prevalent renal transplant patients on 1st January 2013, the death rate during 2013 was 2.4/100 patient years (CI 2.2-2.6) when censored for return to dialysis and 2.5/100 patient years (CI 2.3-2.7) without censoring for dialysis. These death rates are similar to those observed over the last few years and have not shown any impact of the increasing age of the transplanted cohort.

During 2013, 2.4\% of prevalent transplant patients experienced graft failure (excluding death as a cause of graft failure) maintaining the fall in graft failure rates 
Table 3.1. UK kidney and kidney plus other organ transplant numbers in the UK (including paediatric), 1/1/2011-31/12/2013

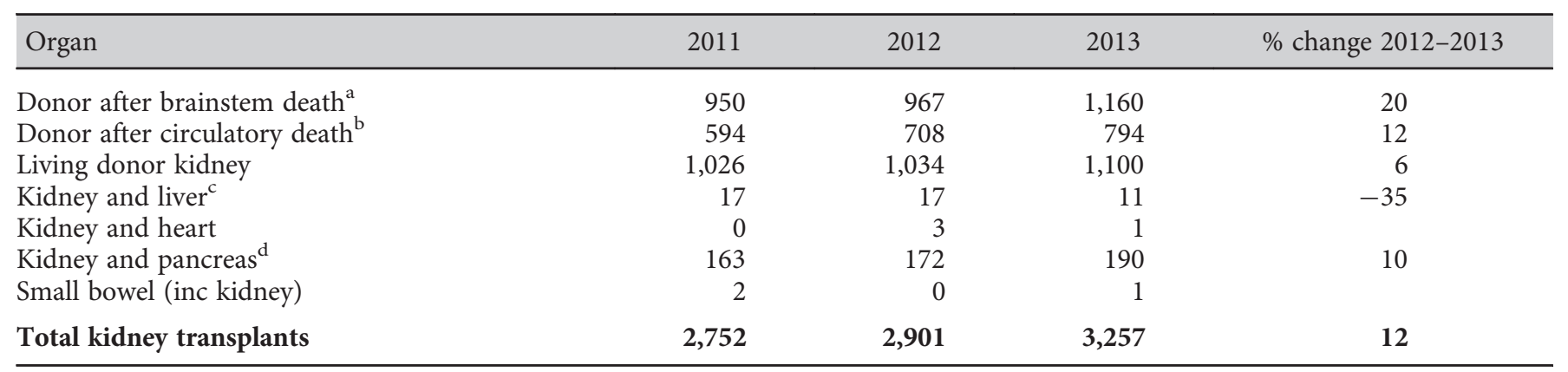

${ }^{a}$ Includes en bloc kidney transplants (7 in 2011, 4 in 2012, 4 in 2013) and double kidney transplants (5 in 2011, 7 in 2012, 18 in 2013)

${ }^{\mathrm{b}}$ Includes en bloc kidney transplants (2 in 2011, 4 in 2012, 6 in 2013) and double kidney transplants (32 in 2011, 52 in 2012,53 in 2013 )

${ }^{\mathrm{c}}$ Includes DCD transplants (2 in 2013)

${ }^{\mathrm{d}}$ Includes DCD transplants (28 in 2011, 35 in 2012, 36 in 2013)

noted over the last couple of years. Whilst it might be premature to assume that graft failure rates are falling in the UK the $0.5 \%$ fall noted in the last five years is certainly encouraging.

\section{Conclusions}

In 2013, the increased number of kidney transplants performed was mostly due to an increase in organs from donors after brainstem death. The graft failure

Table 3.2. Risk-adjusted first adult kidney transplant only, graft and patient survival percentage rates for UK centres ${ }^{a}$

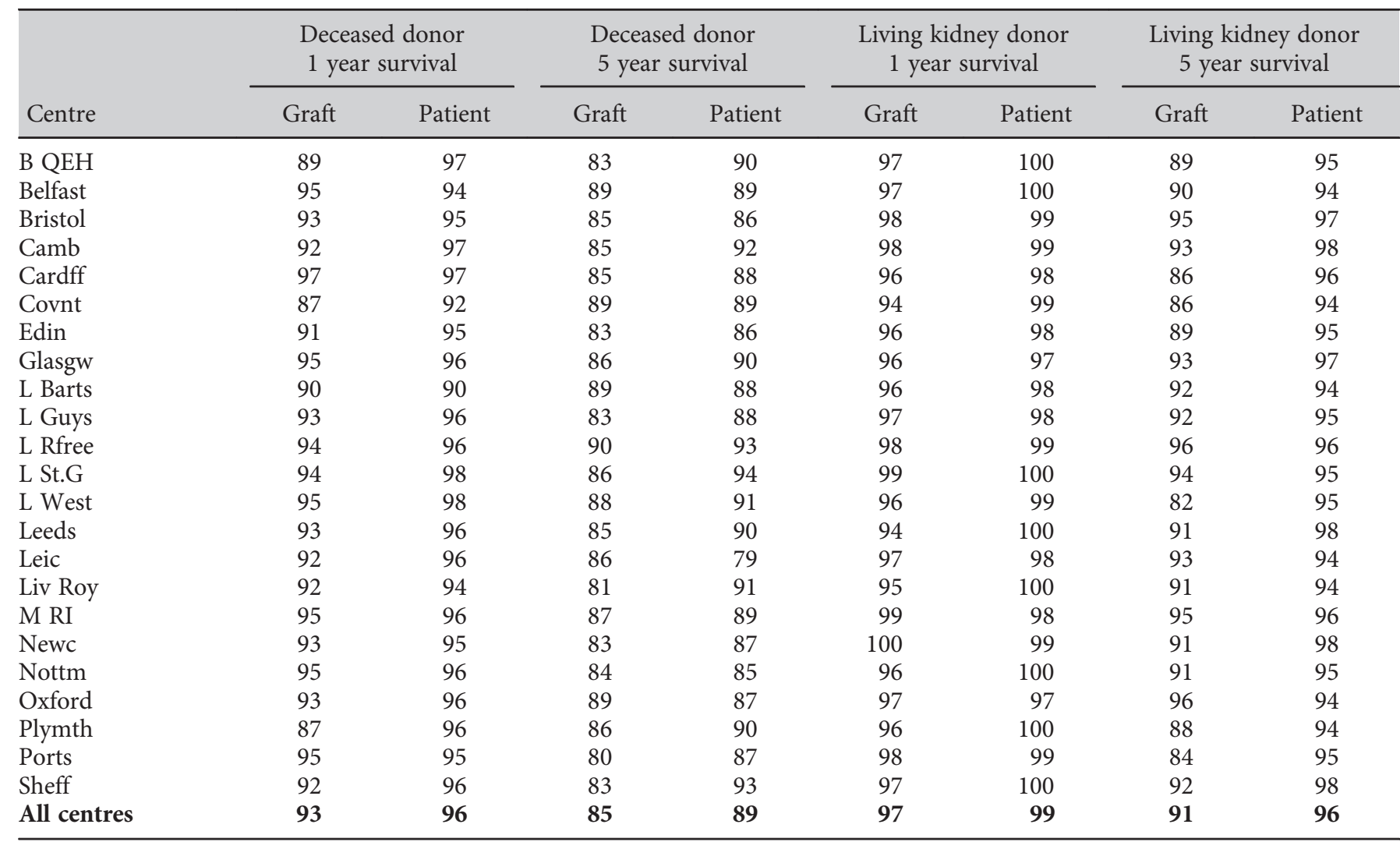

Cohorts for survival rate estimation: 1 year survival: 1/1/2008-31/12/2012; 5 year survival: 1/1/2004-31/12/2008; first grafts only - re-grafts excluded for patient survival estimation. Since the cohorts to estimate 1- and 5-year survival are different, some centres may appear to have 5 year survival better than 1 year survival

${ }^{a}$ Information courtesy of NHSBT: number of transplants, patients and 95\% CI for each estimate; statistical methodology for computing risk-adjusted estimates can be obtained from the NHSBT website (see http://www.organdonation.nhs.uk/ukt/statistics/statistics.asp) 
rate of $2.4 \%$ per annum and patient death rate of 2.4 per 100 patient years were similar to those noted in 2012 .

\section{Transplant demographics}

\section{Introduction}

Since 2008, all UK renal centres have established electronic linkage to the UKRR or Scottish Renal Registry, giving the UKRR complete coverage of individual patient level data across the UK.

The following sections need to be interpreted in the context of variable repatriation policies; some transplant centres continue to follow up and report on all patients they transplant, whereas others refer patients back to nontransplant centres for most or all ongoing post-transplant care. Some transplant centres only refer back patients when their graft is failing. The time post-transplantation that a patient is referred back to their local centre varies between transplant centres. The UKRR is able to detect duplicate patients (being reported from both transplant and referring centres) and in such situations care is usually attributed to the referring centre (see appendix B2 for allocation procedure). This process may result in some discrepancies in transplant numbers particularly in Oxford/Reading and Clywd/Liverpool Royal.

\section{Methods}

Two centres (Bangor and Colchester) did not have any transplant patients and were excluded from some of the analyses. Their dialysis patients were included in the relevant dialysis population denominators.

For the analysis of primary renal diagnosis (PRD) in transplant recipients, a few centres were excluded from some of the take-on years because of concerns relating to the reliability of PRD coding (with these centres submitting a high percentage of uncertain or missing aetiology codes).

Information on patient demographics (age, gender, ethnicity and $\mathrm{PRD}$ ) for patients in a given renal centre was obtained from UKRR patient registration data fields. Individual patients were assigned to the centre that returned data for them during 2013. The prevalence of transplant patients in areas covered by individual Clinical Commissioning Groups (CCG) or Health Board/Social
Care Areas (HB) was estimated based on the postcode of the registered address for patients on renal replacement therapy (RRT). Data on ethnic origin, supplied as Patient Administration System (PAS) codes, were retrieved from fields within renal centre IT systems. For the purpose of this analysis, patients were grouped into Whites, South Asians, Blacks, Others and Unknown. The details of ethnicity regrouping into the above categories are provided in appendix H: Coding http://www.renalreg.org.

\section{Results and Conclusions}

Prevalent transplant numbers across the UK are described in table 3.3.

The prevalence of renal transplant recipients in each CCG/HB in England, Northern Ireland (Health and Social Care Trust Areas), Scotland (Health Boards) and Wales (Local Health Boards) and the proportion of prevalent patients according to modality in the renal centres across the UK is described in tables 3.4 and 3.5 respectively. After standardisation for age and gender, unexplained variability was evident in the prevalence of renal transplant recipients, with some areas having higher than the predicted number of prevalent transplant patients per million population and others lower. There are a number of potential explanations for these inconsistencies, including geographical differences in access to renal transplantation in the UK. This has previously been analysed in detail by the UKRR [2] and is currently the focus of a large national study (access to Transplant and Transplant Outcome Measures (ATTOM)).

The proportion of prevalent RRT patients with a transplant relative to the number on dialysis has been relatively stable over the last decade.

\section{Age and gender}

The gender ratio amongst incident and prevalent transplant patients has remained stable for at least the last ten years (table 3.6, figure 3.1). Note, absolute patient numbers differ from those published in previous reports as a result of additional data validation and reallocation of patients. The average age of incident transplant patients has steadily increased during the same time period. There has also been a gradual increase in the average age of prevalent transplant patients, which

Table 3.3. The prevalence per million population ( $\mathrm{pmp}$ ) of renal transplants in adults in the UK on $31 / 12 / 2013$, by country

\begin{tabular}{lcrrrr}
\hline & England & N Ireland & Scotland & Wales & UK \\
\hline Number of prevalent transplant patients & 24,782 & 815 & 2,478 & 1,517 & 29,592 \\
Total population, mid-2013 estimates from ONS* (millions) & 53.9 & 1.8 & 5.3 & 3.1 & 64.1 \\
Prevalence pmp transplant & 460 & 445 & 465 & 492 & 462 \\
\hline
\end{tabular}

* Office of National Statistics, UK 
Table 3.4. The prevalence per million population ( $\mathrm{pmp}$ ) of patients with a renal transplant and standardised rate ratio in the UK, as on 31st December 2009-2013, by CCG/HB

${ }^{a} \mathrm{CCG} / \mathrm{HB}$ - Clinical Commissioning Group (England); Health and Social Care Trust Areas (Northern Ireland); Health Board (Scotland) and Local Health Board (Wales)

${ }^{\mathrm{b}}$ Population numbers based on the 2012 mid-year estimates by age group and gender (data obtained from the Office of National Statistics, National Records of Scotland and the Northern Ireland Statistics and Research Agency - based on the 2011 Census)

${ }^{\mathrm{c}} \mathrm{O} / \mathrm{E}$ - age and gender standardised prevelence rate ratio

CCG/HBs with significantly high average rate ratios are bold in greyed areas

CCG/HBs with significantly low average rate ratios are italicised in greyed areas

LCL - lower 95\% confidence limit

UCL - upper 95\% confidence limit

$\%$ non-White - percentage of the CCG/HB population that is non-White, from 2011 Census

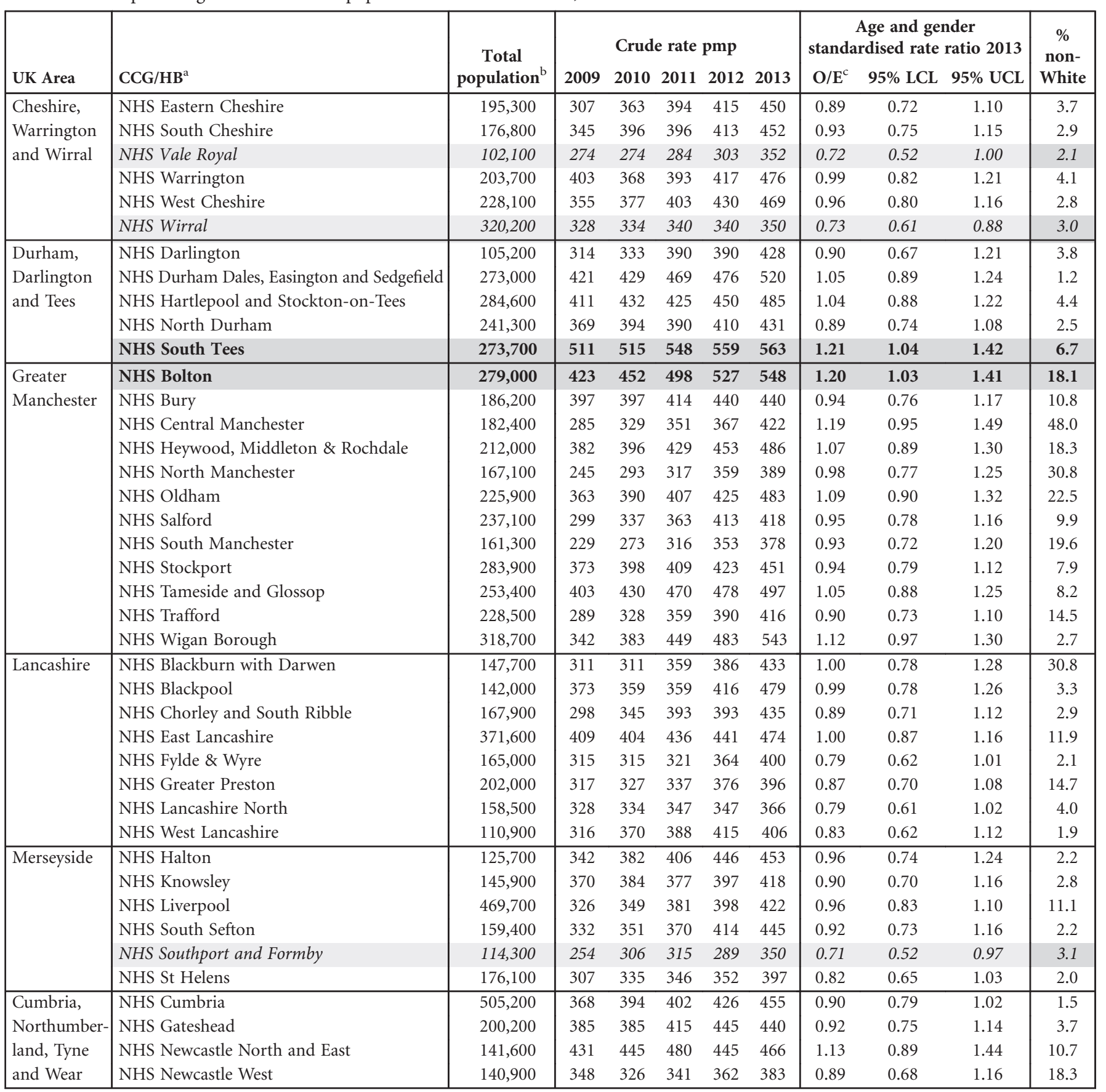


Table 3.4. Continued

\begin{tabular}{|c|c|c|c|c|c|c|c|c|c|c|c|}
\hline \multirow[b]{2}{*}{ UK Area } & \multirow[b]{2}{*}{$\mathrm{CCG} / \mathrm{HB}^{\mathrm{a}}$} & \multirow{2}{*}{$\begin{array}{c}\text { Total } \\
\text { population }^{\mathrm{b}}\end{array}$} & \multicolumn{5}{|c|}{ Crude rate pmp } & \multicolumn{3}{|c|}{$\begin{array}{c}\text { Age and gender } \\
\text { standardised rate ratio } 2013\end{array}$} & \multirow{2}{*}{$\begin{array}{c}\% \\
\text { non- } \\
\text { White }\end{array}$} \\
\hline & & & 2009 & 2010 & 2011 & 2012 & 2013 & $\mathbf{O} / \mathbf{E}^{\mathrm{c}}$ & 95\% LCL & 95\% UCL & \\
\hline \multirow{4}{*}{$\begin{array}{l}\text { Cumbria, } \\
\text { Northumber- } \\
\text { land, Tyne } \\
\text { and Wear }\end{array}$} & NHS North Tyneside & 201,400 & 526 & 571 & 586 & 586 & 586 & 1.21 & 1.01 & 1.44 & 3.4 \\
\hline & NHS Northumberland & 316,100 & 414 & 389 & 437 & 446 & 481 & 0.94 & 0.81 & 1.11 & 1.6 \\
\hline & NHS South Tyneside & 148,400 & 472 & 472 & 505 & 512 & 559 & 1.16 & 0.93 & 1.43 & 4.1 \\
\hline & NHS Sunderland & 275,700 & 417 & 439 & 482 & 497 & 519 & 1.08 & 0.92 & 1.27 & 4.1 \\
\hline \multirow{5}{*}{$\begin{array}{l}\text { North } \\
\text { Yorkshire } \\
\text { and Humber }\end{array}$} & NHS Harrogate and Rural District & 158,600 & 435 & 467 & 479 & 530 & 536 & 1.09 & 0.88 & 1.35 & 3.7 \\
\hline & NHS Hull & 257,200 & 369 & 385 & 404 & 435 & 478 & 1.08 & 0.91 & 1.29 & 5.9 \\
\hline & NHS North East Lincolnshire & 159,700 & 363 & 369 & 419 & 445 & 470 & 1.00 & 0.80 & 1.25 & 2.6 \\
\hline & NHS North Lincolnshire & 168,400 & 267 & 267 & 279 & 291 & 315 & 0.65 & 0.49 & 0.85 & 4.0 \\
\hline & NHS Scarborough and Ryedale & 110,500 & 407 & 425 & 453 & 434 & 425 & 0.85 & 0.64 & 1.13 & 2.5 \\
\hline \multirow{2}{*}{$\begin{array}{l}\text { South } \\
\text { Yorkshire } \\
\text { and } \\
\text { Bassetlaw }\end{array}$} & NHS Rotherham & 258,400 & 356 & 399 & 434 & 457 & 492 & 1.03 & 0.86 & 1.22 & 6.4 \\
\hline & NHS Sheffield & 557,400 & 319 & 355 & 382 & 395 & 418 & 0.96 & 0.85 & 1.10 & 16.3 \\
\hline \multirow{9}{*}{$\begin{array}{l}\text { West } \\
\text { Yorkshire }\end{array}$} & NHS Airedale, Wharfedale and Craven & 158,200 & 417 & 455 & 436 & 449 & 474 & 0.98 & 0.78 & 1.23 & 11.1 \\
\hline & NHS Bradford City & 82,300 & 377 & 389 & 401 & 486 & 522 & 1.56 & 1.16 & 2.10 & 72.2 \\
\hline & NHS Bradford Districts & 333,500 & 429 & 462 & 471 & 525 & 570 & 1.32 & 1.15 & 1.52 & 28.7 \\
\hline & NHS Calderdale & 205,300 & 434 & 472 & 507 & 536 & 531 & 1.11 & 0.92 & 1.34 & 10.3 \\
\hline & NHS Greater Huddersfield & 238,800 & 373 & 398 & 431 & 461 & 473 & 1.01 & 0.84 & 1.22 & 17.4 \\
\hline & NHS Leeds North & 199,600 & 351 & 366 & 406 & 416 & 421 & 0.90 & 0.73 & 1.12 & 17.4 \\
\hline & NHS Leeds South and East & 238,300 & 348 & 378 & 394 & 407 & 466 & 1.09 & 0.90 & 1.31 & 18.3 \\
\hline & NHS Leeds West & 319,800 & 294 & 328 & 350 & 403 & 441 & 1.05 & 0.89 & 1.24 & 10.8 \\
\hline & NHS North Kirklees & 186,700 & 477 & 487 & 509 & 514 & 595 & 1.34 & 1.11 & 1.61 & 25.3 \\
\hline $\begin{array}{l}\text { Arden, } \\
\text { Herefordshire } \\
\text { and } \\
\text { Worcester- } \\
\text { shire }\end{array}$ & NHS Wyre Forest & 98,100 & 357 & 357 & 357 & 377 & 408 & 0.81 & 0.59 & 1.10 & 2.8 \\
\hline \multirow{7}{*}{$\begin{array}{l}\text { Birmingham } \\
\text { and the } \\
\text { Black } \\
\text { Country }\end{array}$} & NHS Birmingham CrossCity & 721,400 & 352 & 371 & 391 & 413 & 438 & 1.06 & 0.95 & 1.19 & 35.2 \\
\hline & NHS Birmingham South and Central & 199,600 & 361 & 381 & 371 & 366 & 431 & 1.09 & 0.88 & 1.34 & 40.4 \\
\hline & NHS Dudley & 313,600 & 297 & 303 & 309 & 290 & 328 & 0.69 & 0.57 & 0.84 & 10.0 \\
\hline & NHS Sandwell and West Birmingham & 475,700 & 349 & 357 & 368 & 397 & 454 & 1.10 & 0.96 & 1.26 & 45.3 \\
\hline & NHS Solihull & 207,400 & 285 & 299 & 313 & 338 & 342 & 0.72 & 0.57 & 0.91 & 10.9 \\
\hline & NHS Walsall & 270,900 & 376 & 388 & 413 & 428 & 469 & 1.04 & 0.87 & 1.24 & 21.1 \\
\hline & NHS Wolverhampton & 251,000 & 299 & 303 & 299 & 315 & 379 & 0.85 & 0.70 & 1.04 & 32.0 \\
\hline \multirow{10}{*}{$\begin{array}{l}\text { Derbyshire } \\
\text { and } \\
\text { Nottingham- } \\
\text { shire }\end{array}$} & NHS Erewash & 94,600 & 264 & 285 & 285 & 296 & 412 & 0.86 & 0.63 & 1.18 & 3.2 \\
\hline & NHS Hardwick & 108,900 & 257 & 266 & 257 & 257 & 257 & 0.52 & 0.36 & 0.75 & 1.8 \\
\hline & NHS Mansfield \& Ashfield & 192,500 & 322 & 358 & 400 & 452 & 473 & 0.98 & 0.80 & 1.20 & 2.5 \\
\hline & NHS Newark \& Sherwood & 115,900 & 380 & 431 & 440 & 492 & 535 & 1.08 & 0.84 & 1.39 & 2.4 \\
\hline & NHS North Derbyshire & 272,100 & 320 & 334 & 364 & 404 & 401 & 0.79 & 0.66 & 0.96 & 2.5 \\
\hline & NHS Nottingham City & 308,700 & 233 & 314 & 330 & 353 & 395 & 1.00 & 0.84 & 1.19 & 28.5 \\
\hline & NHS Nottingham North \& East & 146,200 & 301 & 342 & 383 & 410 & 438 & 0.90 & 0.70 & 1.15 & 6.2 \\
\hline & NHS Nottingham West & 110,700 & 379 & 443 & 461 & 470 & 533 & 1.09 & 0.85 & 1.41 & 7.3 \\
\hline & NHS Rushcliffe & 111,600 & 332 & 341 & 385 & 403 & 457 & 0.93 & 0.71 & 1.23 & 6.9 \\
\hline & NHS Southern Derbyshire & 515,300 & 311 & 357 & 390 & 411 & 444 & 0.95 & 0.84 & 1.08 & 11.0 \\
\hline
\end{tabular}


Table 3.4. Continued

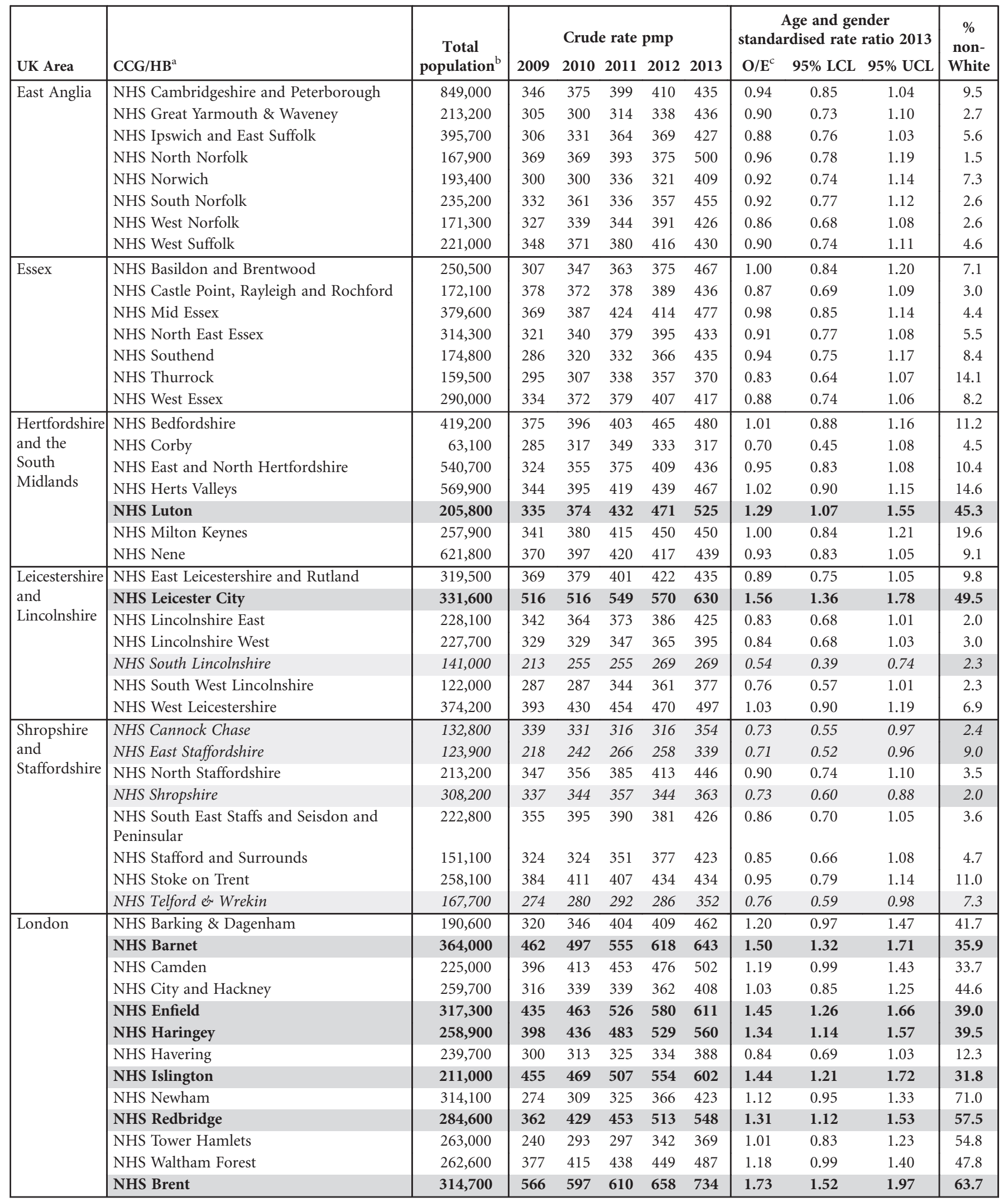


Table 3.4. Continued

\begin{tabular}{|c|c|c|c|c|c|c|c|c|c|c|c|}
\hline \multirow[b]{2}{*}{ UK Area } & \multirow[b]{2}{*}{$\mathrm{CCG} / \mathrm{HB}^{\mathrm{a}}$} & \multirow{2}{*}{$\begin{array}{c}\text { Total } \\
\text { population }^{\mathrm{b}}\end{array}$} & \multicolumn{5}{|c|}{ Crude rate pmp } & \multicolumn{3}{|c|}{$\begin{array}{c}\text { Age and gender } \\
\text { standardised rate ratio } 2013\end{array}$} & \multirow{2}{*}{$\begin{array}{c}\% \\
\text { non- } \\
\text { White }\end{array}$} \\
\hline & & & 2009 & 2010 & 2011 & 2012 & 2013 & $\mathbf{O} / \mathbf{E}^{\mathrm{c}}$ & $95 \% \mathrm{LCL}$ & $95 \%$ UCL & \\
\hline \multirow[t]{19}{*}{ London } & NHS Central London (Westminster) & 161,000 & 391 & 435 & 428 & 466 & 503 & 1.11 & 0.89 & 1.38 & 36.2 \\
\hline & NHS Ealing & 340,700 & 543 & 578 & 596 & 628 & 643 & 1.50 & 1.31 & 1.71 & 51.0 \\
\hline & NHS Hammersmith and Fulham & 179,900 & 400 & 434 & 428 & 450 & 484 & 1.14 & 0.93 & 1.41 & 31.9 \\
\hline & NHS Harrow & 242,400 & 656 & 710 & 710 & 734 & 747 & 1.69 & 1.46 & 1.95 & 57.8 \\
\hline & NHS Hillingdon & 281,800 & 472 & 518 & 568 & 596 & 600 & 1.40 & 1.21 & 1.63 & 39.4 \\
\hline & NHS Hounslow & 259,100 & 471 & 510 & 525 & 548 & 618 & 1.45 & 1.24 & 1.69 & 48.6 \\
\hline & $\begin{array}{l}\text { NHS West London (Kensington and } \\
\text { Chelsea, Queen's Park and Paddington) }\end{array}$ & 218,800 & 421 & 494 & 494 & 498 & 507 & 1.11 & 0.93 & 1.34 & 33.4 \\
\hline & NHS Bexley & 234,300 & 465 & 516 & 529 & 538 & 581 & 1.29 & 1.09 & 1.53 & 18.1 \\
\hline & NHS Bromley & 314,000 & 452 & 494 & 494 & 516 & 541 & 1.17 & 1.00 & 1.36 & 15.7 \\
\hline & NHS Croydon & 368,900 & 317 & 331 & 358 & 377 & 412 & 0.95 & 0.81 & 1.11 & 44.9 \\
\hline & NHS Greenwich & 260,100 & 342 & 369 & 404 & 446 & 481 & 1.16 & 0.97 & 1.38 & 37.5 \\
\hline & NHS Kingston & 163,900 & 390 & 397 & 409 & 451 & 470 & 1.07 & 0.86 & 1.34 & 25.5 \\
\hline & NHS Lambeth & 310,200 & 326 & 335 & 374 & 416 & 464 & 1.13 & 0.96 & 1.33 & 42.9 \\
\hline & NHS Lewisham & 281,600 & 391 & 380 & 391 & 408 & 497 & 1.19 & 1.00 & 1.40 & 46.5 \\
\hline & NHS Merton & 202,200 & 405 & 415 & 455 & 499 & 559 & 1.28 & 1.07 & 1.54 & 35.1 \\
\hline & NHS Richmond & 189,100 & 291 & 307 & 333 & 360 & 391 & 0.84 & 0.67 & 1.06 & 14.0 \\
\hline & NHS Southwark & 293,500 & 453 & 480 & 511 & 555 & 596 & 1.44 & 1.24 & 1.67 & 45.8 \\
\hline & NHS Sutton & 193,600 & 418 & 444 & 454 & 491 & 511 & 1.12 & 0.92 & 1.37 & 21.4 \\
\hline & NHS Wandsworth & 308,300 & 318 & 334 & 373 & 405 & 441 & 1.07 & 0.90 & 1.26 & 28.6 \\
\hline \multirow{4}{*}{$\begin{array}{l}\text { Bath, Glou- } \\
\text { cestershire, } \\
\text { Swindon and } \\
\text { Wiltshire }\end{array}$} & NHS Bath and North East Somerset & 177,600 & 304 & 293 & 287 & 293 & 360 & 0.79 & 0.62 & 1.01 & 5.4 \\
\hline & NHS Gloucestershire & 602,200 & 345 & 354 & 384 & 380 & 425 & 0.88 & 0.78 & 0.99 & 4.6 \\
\hline & NHS Swindon & 217,200 & 350 & 414 & 437 & 447 & 488 & 1.05 & 0.87 & 1.27 & 10.0 \\
\hline & NHS Wiltshire & 476,800 & 323 & 354 & 382 & 394 & 398 & 0.83 & 0.72 & 0.95 & 3.4 \\
\hline \multirow{4}{*}{$\begin{array}{l}\text { Bristol, North } \\
\text { Somerset, } \\
\text { Somerset and } \\
\text { South Glou- } \\
\text { cestershire } \\
\end{array}$} & NHS Bristol & 432,500 & 451 & 476 & 486 & 511 & 550 & 1.31 & 1.15 & 1.49 & 16.0 \\
\hline & NHS North Somerset & 204,400 & 396 & 421 & 431 & 465 & 484 & 0.98 & 0.81 & 1.20 & 2.7 \\
\hline & NHS Somerset & 535,000 & 363 & 383 & 413 & 415 & 436 & 0.89 & 0.78 & 1.01 & 2.0 \\
\hline & NHS South Gloucestershire & 266,100 & 436 & 455 & 466 & 481 & 507 & 1.07 & 0.90 & 1.27 & 5.0 \\
\hline \multirow{3}{*}{$\begin{array}{l}\text { Devon, } \\
\text { Cornwall and } \\
\text { Isles of Scilly }\end{array}$} & NHS Kernow & 540,200 & 441 & 452 & 476 & 515 & 546 & 1.09 & 0.97 & 1.22 & 1.8 \\
\hline & NHS North, East, West Devon & 869,400 & 428 & 435 & 444 & 468 & 504 & 1.05 & 0.96 & 1.15 & 3.0 \\
\hline & NHS South Devon and Torbay & 273,300 & 428 & 461 & 483 & 487 & 545 & 1.08 & 0.92 & 1.27 & 2.1 \\
\hline \multirow{8}{*}{$\begin{array}{l}\text { Kent and } \\
\text { Medway }\end{array}$} & NHS Ashford & 120,100 & 425 & 441 & 466 & 516 & 516 & 1.11 & 0.86 & 1.42 & 6.3 \\
\hline & NHS Canterbury and Coastal & 200,300 & 374 & 399 & 419 & 479 & 494 & 1.09 & 0.89 & 1.32 & 5.9 \\
\hline & NHS Dartford, Gravesham and Swanley & 249,200 & 465 & 474 & 457 & 478 & 510 & 1.11 & 0.93 & 1.32 & 13.0 \\
\hline & NHS Medway & 268,200 & 380 & 406 & 414 & 447 & 492 & 1.08 & 0.91 & 1.29 & 10.4 \\
\hline & NHS South Kent Coast & 203,000 & 310 & 345 & 374 & 394 & 419 & 0.86 & 0.69 & 1.06 & 4.5 \\
\hline & NHS Swale & 108,200 & 416 & 425 & 517 & 545 & 601 & 1.28 & 1.00 & 1.63 & 3.8 \\
\hline & NHS Thanet & 135,700 & 361 & 405 & 450 & 538 & 597 & 1.27 & 1.02 & 1.58 & 4.5 \\
\hline & NHS West Kent & 463,700 & 354 & 358 & 377 & 403 & 421 & 0.89 & 0.77 & 1.02 & 4.9 \\
\hline \multirow{12}{*}{$\begin{array}{l}\text { Surrey and } \\
\text { Sussex }\end{array}$} & NHS Brighton \& Hove & 275,800 & 305 & 348 & 363 & 370 & 388 & 0.88 & 0.73 & 1.06 & 10.9 \\
\hline & NHS Coastal West Sussex & 476,700 & 386 & 399 & 424 & 424 & 462 & 0.94 & 0.83 & 1.08 & 3.8 \\
\hline & NHS Crawley & 108,300 & 286 & 286 & 314 & 332 & 342 & 0.78 & 0.57 & 1.08 & 20.1 \\
\hline & NHS East Surrey & 175,900 & 313 & 313 & 318 & 335 & 370 & 0.78 & 0.62 & 1.00 & 8.3 \\
\hline & NHS Eastbourne, Hailsham and Seaford & 182,000 & 302 & 319 & 330 & 346 & 368 & 0.77 & 0.60 & 0.98 & 4.4 \\
\hline & NHS Guildford and Waverley & 205,900 & 306 & 301 & 291 & 345 & 354 & 0.77 & 0.61 & 0.97 & 7.2 \\
\hline & NHS Hastings \& Rother & 181,400 & 303 & 325 & 353 & 342 & 364 & 0.74 & 0.58 & 0.94 & 4.6 \\
\hline & NHS High Weald Lewes Havens & 167,800 & 328 & 334 & 352 & 411 & 417 & 0.83 & 0.66 & 1.05 & 3.1 \\
\hline & NHS Horsham and Mid Sussex & 223,300 & 313 & 331 & 336 & 336 & 367 & 0.76 & 0.61 & 0.95 & 4.9 \\
\hline & NHS North West Surrey & 338,200 & 411 & 417 & 423 & 444 & 467 & 0.99 & 0.85 & 1.16 & 12.5 \\
\hline & NHS Surrey Downs & 282,700 & 368 & 389 & 393 & 407 & 432 & 0.89 & 0.75 & 1.07 & 9.1 \\
\hline & NHS Surrey Heath & 94,100 & 446 & 468 & 489 & 521 & 499 & 1.03 & 0.78 & 1.38 & 9.3 \\
\hline
\end{tabular}


Table 3.4. Continued

\begin{tabular}{|c|c|c|c|c|c|c|c|c|c|c|c|}
\hline \multirow[b]{2}{*}{ UK Area } & \multirow[b]{2}{*}{$\mathrm{CCG} / \mathrm{HB}^{\mathrm{a}}$} & \multirow{2}{*}{$\begin{array}{c}\text { Total } \\
\text { population }\end{array}$} & \multicolumn{5}{|c|}{ Crude rate pmp } & \multicolumn{3}{|c|}{$\begin{array}{c}\text { Age and gender } \\
\text { standardised rate ratio } 2013\end{array}$} & \multirow{2}{*}{$\begin{array}{c}\% \\
\text { non- } \\
\text { White }\end{array}$} \\
\hline & & & 2009 & 2010 & 2011 & 2012 & 2013 & $\mathbf{O} / \mathbf{E}^{\mathrm{c}}$ & 95\% LCL & 95\% UCL & \\
\hline \multirow{10}{*}{$\begin{array}{l}\text { Thames } \\
\text { Valley }\end{array}$} & NHS Aylesbury Vale & 196,400 & 484 & 494 & 525 & 545 & 555 & 1.16 & 0.96 & 1.40 & 9.7 \\
\hline & NHS Bracknell and Ascot & 132,900 & 376 & 414 & 451 & 481 & 504 & 1.09 & 0.86 & 1.38 & 9.5 \\
\hline & NHS Chiltern & 317,900 & 396 & 425 & 425 & 469 & 497 & 1.05 & 0.90 & 1.23 & 15.8 \\
\hline & NHS Newbury and District & 105,100 & 561 & 542 & 618 & 618 & 628 & 1.32 & 1.03 & 1.67 & 4.4 \\
\hline & NHS North \& West Reading & 99,300 & 332 & 403 & 403 & 433 & 493 & 1.04 & 0.78 & 1.37 & 10.4 \\
\hline & NHS Oxfordshire & 647,100 & 400 & 423 & 436 & 473 & 487 & 1.06 & 0.95 & 1.19 & 9.3 \\
\hline & NHS Slough & 141,800 & 585 & 642 & 649 & 684 & 818 & 2.02 & 1.69 & 2.43 & 54.3 \\
\hline & NHS South Reading & 107,200 & 560 & 560 & 579 & 569 & 607 & 1.52 & 1.19 & 1.94 & 30.5 \\
\hline & NHS Windsor, Ascot and Maidenhead & 139,000 & 338 & 410 & 439 & 511 & 561 & 1.22 & 0.98 & 1.53 & 14.7 \\
\hline & NHS Wokingham & 156,700 & 389 & 389 & 402 & 434 & 440 & 0.92 & 0.73 & 1.17 & 11.6 \\
\hline \multirow[t]{9}{*}{ Wessex } & NHS Dorset & 750,300 & 396 & 407 & 415 & 409 & 425 & 0.87 & 0.78 & 0.98 & 4.0 \\
\hline & NHS Fareham and Gosport & 196,100 & 403 & 403 & 418 & 423 & 479 & 0.99 & 0.81 & 1.21 & 3.4 \\
\hline & NHS Isle of Wight & 138,700 & 346 & 360 & 368 & 382 & 375 & 0.74 & 0.57 & 0.97 & 2.7 \\
\hline & NHS North East Hampshire and Farnham & 206,800 & 329 & 368 & 368 & 387 & 416 & 0.89 & 0.72 & 1.11 & 9.7 \\
\hline & NHS North Hampshire & 216,200 & 314 & 328 & 356 & 370 & 379 & 0.79 & 0.64 & 0.99 & 6.4 \\
\hline & NHS Portsmouth & 206,800 & 348 & 396 & 392 & 406 & 435 & 1.03 & 0.84 & 1.26 & 11.6 \\
\hline & NHS South Eastern Hampshire & 209,100 & 387 & 416 & 411 & 445 & 459 & 0.94 & 0.77 & 1.15 & 3.1 \\
\hline & NHS Southampton & 239,400 & 338 & 338 & 384 & 418 & 464 & 1.12 & 0.93 & 1.35 & 14.1 \\
\hline & NHS West Hampshire & 544,400 & 373 & 393 & 406 & 417 & 435 & 0.89 & 0.78 & 1.01 & 3.9 \\
\hline \multirow[t]{7}{*}{ Wales } & Betsi Cadwaladr University & 690,400 & 343 & 359 & 359 & 352 & 336 & 0.69 & 0.61 & 0.79 & 2.5 \\
\hline & Powys Teaching & 133,000 & 361 & 399 & 391 & 354 & 369 & 0.72 & 0.55 & 0.95 & 1.6 \\
\hline & Hywel Dda & 383,400 & 412 & 409 & 438 & 436 & 503 & 1.03 & 0.90 & 1.19 & 2.2 \\
\hline & Abertawe Bro Morgannwg University & 519,500 & 449 & 485 & 541 & 576 & 603 & 1.27 & 1.14 & 1.42 & 3.9 \\
\hline & Cwm Taf & 294,500 & 567 & 628 & 662 & 686 & 740 & 1.58 & 1.38 & 1.80 & 2.6 \\
\hline & Aneurin Bevan & 578,000 & 469 & 500 & 523 & 587 & 599 & 1.26 & 1.14 & 1.40 & 3.9 \\
\hline & Cardiff and Vale University & 475,300 & 406 & 440 & 467 & 501 & 515 & 1.18 & 1.04 & 1.34 & 12.2 \\
\hline \multirow[t]{14}{*}{ Scotland } & Ayrshire and Arran & 373,200 & 386 & 383 & 375 & 402 & 426 & 0.86 & 0.73 & 1.00 & 1.2 \\
\hline & Borders & 113,700 & 361 & 413 & 413 & 466 & 484 & 0.93 & 0.72 & 1.22 & 1.3 \\
\hline & Dumfries and Galloway & 150,800 & 351 & 345 & 371 & 365 & 378 & 0.74 & 0.57 & 0.95 & 1.2 \\
\hline & Fife & 366,200 & 306 & 319 & 344 & 355 & 388 & 0.80 & 0.68 & 0.94 & 2.4 \\
\hline & Forth Valley & 299,100 & 294 & 311 & 334 & 364 & 395 & 0.81 & 0.68 & 0.97 & 2.2 \\
\hline & Grampian & 573,400 & 351 & 359 & 373 & 398 & 427 & 0.89 & 0.78 & 1.01 & 4.0 \\
\hline & Greater Glasgow and Clyde & $1,217,000$ & 412 & 423 & 440 & 487 & 522 & 1.11 & 1.03 & 1.20 & 7.3 \\
\hline & Highland & 319,800 & 450 & 472 & 463 & 466 & 485 & 0.95 & 0.81 & 1.11 & 1.3 \\
\hline & Lanarkshire & 572,500 & 384 & 402 & 423 & 459 & 479 & 0.99 & 0.88 & 1.11 & 2.0 \\
\hline & Lothian & 843,700 & 316 & 333 & 351 & 361 & 370 & 0.80 & 0.71 & 0.89 & 5.6 \\
\hline & Orkney & 21,500 & 418 & 372 & 372 & 372 & 372 & 0.72 & 0.36 & 1.44 & 0.7 \\
\hline & Shetland & 23,200 & 259 & 259 & 215 & 259 & 259 & 0.52 & 0.24 & 1.16 & 1.5 \\
\hline & Tayside & 411,700 & 398 & 401 & 415 & 425 & 447 & 0.93 & 0.80 & 1.07 & 3.2 \\
\hline & Western Isles & 27,600 & 254 & 254 & 290 & 290 & 327 & 0.63 & 0.33 & 1.21 & 0.9 \\
\hline \multirow{5}{*}{$\begin{array}{l}\text { Northern } \\
\text { Ireland }\end{array}$} & Belfast & 348,300 & 359 & 393 & 405 & 434 & 465 & 1.07 & 0.92 & 1.25 & 3.2 \\
\hline & Northern & 465,500 & 335 & 352 & 367 & 378 & 410 & 0.90 & 0.78 & 1.04 & 1.2 \\
\hline & Southern & 363,100 & 286 & 303 & 341 & 386 & 416 & 0.96 & 0.81 & 1.12 & 1.2 \\
\hline & South Eastern & 350,100 & 363 & 360 & 388 & 394 & 426 & 0.92 & 0.78 & 1.08 & 1.3 \\
\hline & Western & 296,600 & 324 & 344 & 351 & 354 & 438 & 0.99 & 0.83 & 1.17 & 1.0 \\
\hline
\end{tabular}

could reflect the increasing age at which patients are transplanted and/or improved survival after renal transplantation over the last few years. The prevalent transplant patient workload across the UK increased to
29,592 patients at the end of 2013. The continued expansion of this patient group means there is a need for careful planning by renal centres for future service provision and resource allocation. 
Table 3.5. Distribution of prevalent patients on RRT by centre and modality on $31 / 12 / 2013$

\begin{tabular}{|c|c|c|c|c|}
\hline Centre & $N$ & $\% \mathrm{HD}$ & $\% \mathrm{PD}$ & $\%$ Transplant \\
\hline \multicolumn{5}{|c|}{ Transplant centres } \\
\hline B QEH & 2,051 & 45 & 7 & 48 \\
\hline Bristol & 1,427 & 36 & 5 & 59 \\
\hline Camb & 1,198 & 32 & 2 & 66 \\
\hline Cardff & 1,584 & 31 & 5 & 65 \\
\hline Glasgw & 1,598 & 37 & 3 & 60 \\
\hline L Barts & 2,103 & 45 & 9 & 45 \\
\hline L Guys & 1,841 & 34 & 2 & 64 \\
\hline L Rfree & 1,955 & 37 & 7 & 56 \\
\hline L St.G & 759 & 37 & 6 & 57 \\
\hline L West & 3,142 & 44 & 2 & 54 \\
\hline Newc & 964 & 28 & 4 & 67 \\
\hline Nottm & 1,075 & 35 & 8 & 58 \\
\hline Oxford & 1,565 & 28 & 6 & 66 \\
\hline Plymth & 503 & 27 & 7 & 66 \\
\hline Ports & 1,555 & 39 & 5 & 56 \\
\hline Sheff & 1,329 & 44 & 5 & 50 \\
\hline \multicolumn{5}{|c|}{ Dialysis centres } \\
\hline Abrdn & 519 & 43 & 5 & 52 \\
\hline Airdrie & 393 & 49 & 4 & 48 \\
\hline Antrim & 224 & 57 & 7 & 37 \\
\hline B Heart & 658 & 66 & 6 & 28 \\
\hline Clwyd & 153 & 50 & 9 & 41 \\
\hline Colchr & 115 & 100 & & \\
\hline D \& Gall & 117 & 38 & 13 & 49 \\
\hline Derby & 472 & 46 & 18 & 36 \\
\hline Donc & 259 & 63 & 14 & 24 \\
\hline Dorset & 628 & 43 & 8 & 50 \\
\hline Dudley & 312 & 56 & 18 & 26 \\
\hline Dundee & 403 & 43 & 5 & 52 \\
\hline Exeter & 896 & 46 & 8 & 46 \\
\hline Glouc & 412 & 51 & 8 & 41 \\
\hline Hull & 815 & 40 & 10 & 50 \\
\hline Inverns & 216 & 32 & 7 & 61 \\
\hline Ipswi & 354 & 34 & 8 & 57 \\
\hline Kent & 965 & 41 & 7 & 52 \\
\hline Klmarnk & 296 & 46 & 15 & 39 \\
\hline Krkcldy & 283 & 52 & 7 & 41 \\
\hline L Kings & 965 & 52 & 11 & 38 \\
\hline Liv Ain & 190 & 82 & 16 & 3 \\
\hline Middlbr & 836 & 42 & 2 & 56 \\
\hline Newry & 199 & 46 & 9 & 45 \\
\hline
\end{tabular}


Table 3.5. Continued

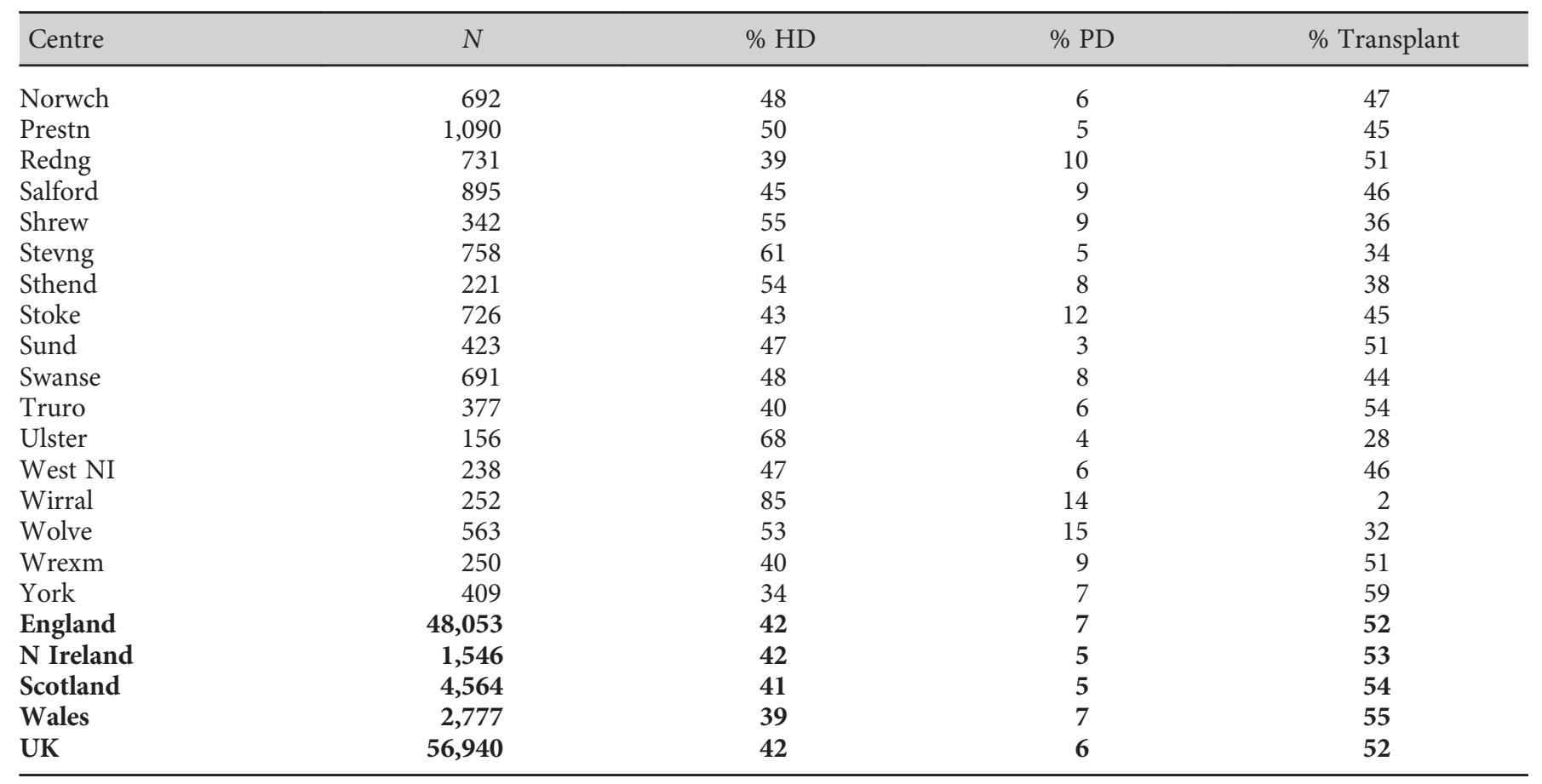

Blank cells: no patients on that modality

Table 3.6. Median age and gender ratio of incident and prevalent transplant patients 2008-2013

\begin{tabular}{|c|c|c|c|c|c|c|}
\hline \multirow[b]{2}{*}{ Year } & \multicolumn{3}{|c|}{ Incident transplants } & \multicolumn{3}{|c|}{ Prevalent transplants ${ }^{*}$} \\
\hline & $N$ & Median age & $\mathrm{M}: \mathrm{F}$ ratio & $N$ & Median age & $\mathrm{M}: \mathrm{F}$ ratio \\
\hline 2009 & 2,496 & 48.3 & 1.6 & 23,508 & 50.8 & 1.5 \\
\hline 2010 & 2,585 & 49.6 & 1.7 & 24,903 & 51.2 & 1.6 \\
\hline 2011 & 2,633 & 49.1 & 1.7 & 26,197 & 51.7 & 1.6 \\
\hline
\end{tabular}

${ }^{*}$ As on 31 st December for given year

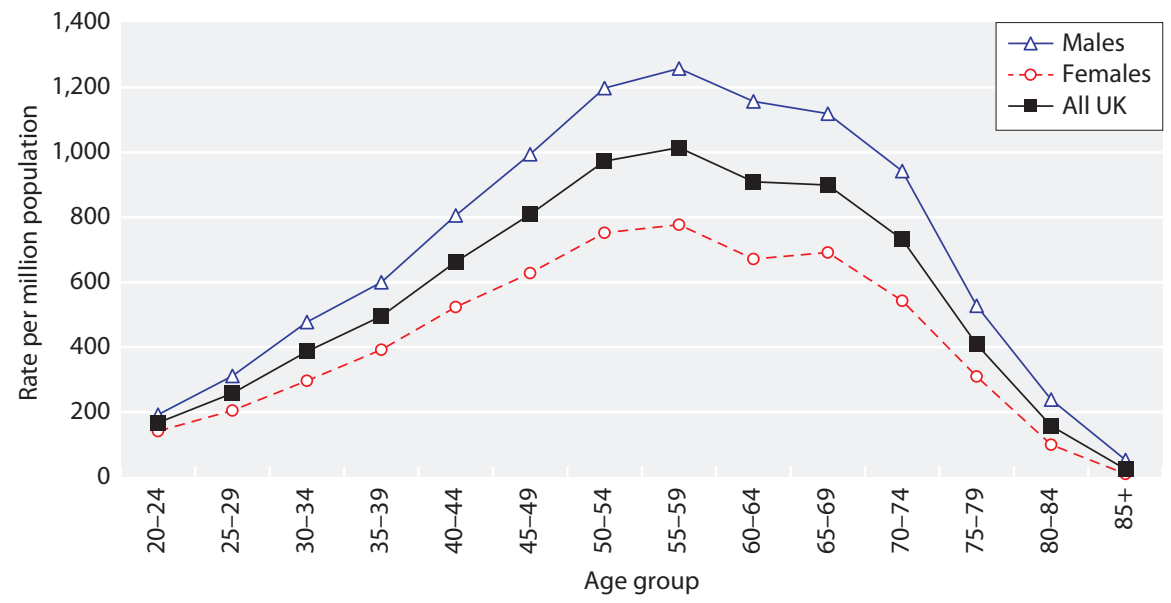

Fig. 3.1. Transplant prevalence rate per million population by age and gender on $31 / 12 / 2013$ 
Table 3.7. Primary renal diagnosis in renal transplant recipients 2008-2013

\begin{tabular}{|c|c|c|c|c|c|c|c|c|c|}
\hline \multirow[b]{3}{*}{ Primary diagnosis } & \multicolumn{7}{|c|}{ New transplants by year } & \multicolumn{2}{|c|}{ Established transplants on $01 / 01 / 2013$} \\
\hline & 2008 & 2009 & 2010 & 2011 & 2012 & & & & \\
\hline & $\%$ & $\%$ & $\%$ & $\%$ & $\%$ & $\%$ & $N$ & $\%$ & $N$ \\
\hline Aetiology uncertain & 14.6 & 14.1 & 14.2 & 14.7 & 11.9 & 12.3 & 374 & 15.4 & 4,243 \\
\hline Diabetes & 13.1 & 13.3 & 12.5 & 13.0 & 15.2 & 13.3 & 407 & 9.9 & 2,737 \\
\hline Glomerulonephritis & 21.9 & 23.5 & 20.4 & 23.1 & 22.6 & 22.3 & 680 & 23.4 & 6,460 \\
\hline Polycystic kidney disease & 13.4 & 13.4 & 14.0 & 12.5 & 13.3 & 13.7 & 419 & 12.9 & 3,555 \\
\hline Pyelonephritis & 12.1 & 11.4 & 10.2 & 10.1 & 10.0 & 9.9 & 302 & 13.5 & 3,733 \\
\hline Reno-vascular disease & 6.7 & 6.2 & 7.3 & 6.5 & 7.0 & 8.1 & 247 & 5.7 & 1,582 \\
\hline Other & 16.8 & 15.7 & 16.4 & 17.2 & 17.5 & 16.0 & 488 & 17.3 & 4,769 \\
\hline Not available & 1.4 & 2.4 & 5.0 & 3.0 & 2.5 & 4.3 & 132 & 1.9 & 526 \\
\hline
\end{tabular}

\section{Primary renal diagnosis}

The primary renal diagnosis of patients receiving kidney transplants in the UK has remained relatively stable over the last five years (table 3.7).

\section{Ethnicity}

It was difficult to compare the proportion of patients within each ethnic group receiving a transplant to those commencing dialysis from the same group because data on ethnicity were missing in a considerable number of patients who were classified as ethnicity 'unknown' (table 3.8). The percentages of patients with unknown ethnicity between 2008 and 2013 provided in this year's chapter are different from those in last year's chapter [3]; this reflects retrospective input of ethnicity data, improving data completeness.

\section{Clinical and laboratory outcomes}

\section{Introduction}

There continued to be marked variation in the completeness of data (tables 3.9a, 3.9b) reported by each renal centre, particularly for blood pressure. Better data records (or possibly better extraction of data held within renal IT systems) would facilitate more meaningful comparisons between centres and help to determine the causes of inter-centre differences in outcomes. For this reason, along with differences in repatriation policies of prevalent transplant patients between centres as highlighted previously, caution needs to be exercised when comparing centre performance.

The 71 renal centres in the UK comprise 52 centres in England, five in Wales, five in Northern Ireland and nine in Scotland. Two centres (Bangor and Colchester) were reported as having no transplanted patients and were therefore excluded. After exclusion of these two centres, prevalent patient data from 69 renal centres across the UK were analysed.

For the one year post-transplant analyses, in which patients were assigned to the centre that performed their transplant, all 23 transplant centres across the UK were included in the analysis.

\section{Methods}

Data for key laboratory variables are reported for all prevalent patients with valid data returns for a given renal centre (both transplanting and non-transplanting centres) and for one year posttransplant results for patients transplanted 2006-2012, with patients attributed to the transplant centre that performed the procedure.

Table 3.8. Ethnicity of patients who received a transplant in the years 2008-2013

\begin{tabular}{lccccc}
\hline Year & \% White & \% S Asian & \% Black & \% Other & \% Unknown \\
\hline 2008 & 76.2 & 9.0 & 6.2 & 1.9 & 6.6 \\
2009 & 74.6 & 10.5 & 6.8 & 2.2 & 6.0 \\
2010 & 75.2 & 10.5 & 5.8 & 2.3 & 2.6 \\
2011 & 74.8 & 9.7 & 7.2 & 3.0 & 6.7 \\
2012 & 72.3 & 9.9 & 7.5 & 2.0 & 8.6 \\
2013 & 70.0 & 12.3 & & & 8.1 \\
\hline
\end{tabular}


Table 3.9a. Percentage completeness of ethnicity, eGFR and blood pressure by centre for prevalent transplant patients on $31 / 12 / 2013$

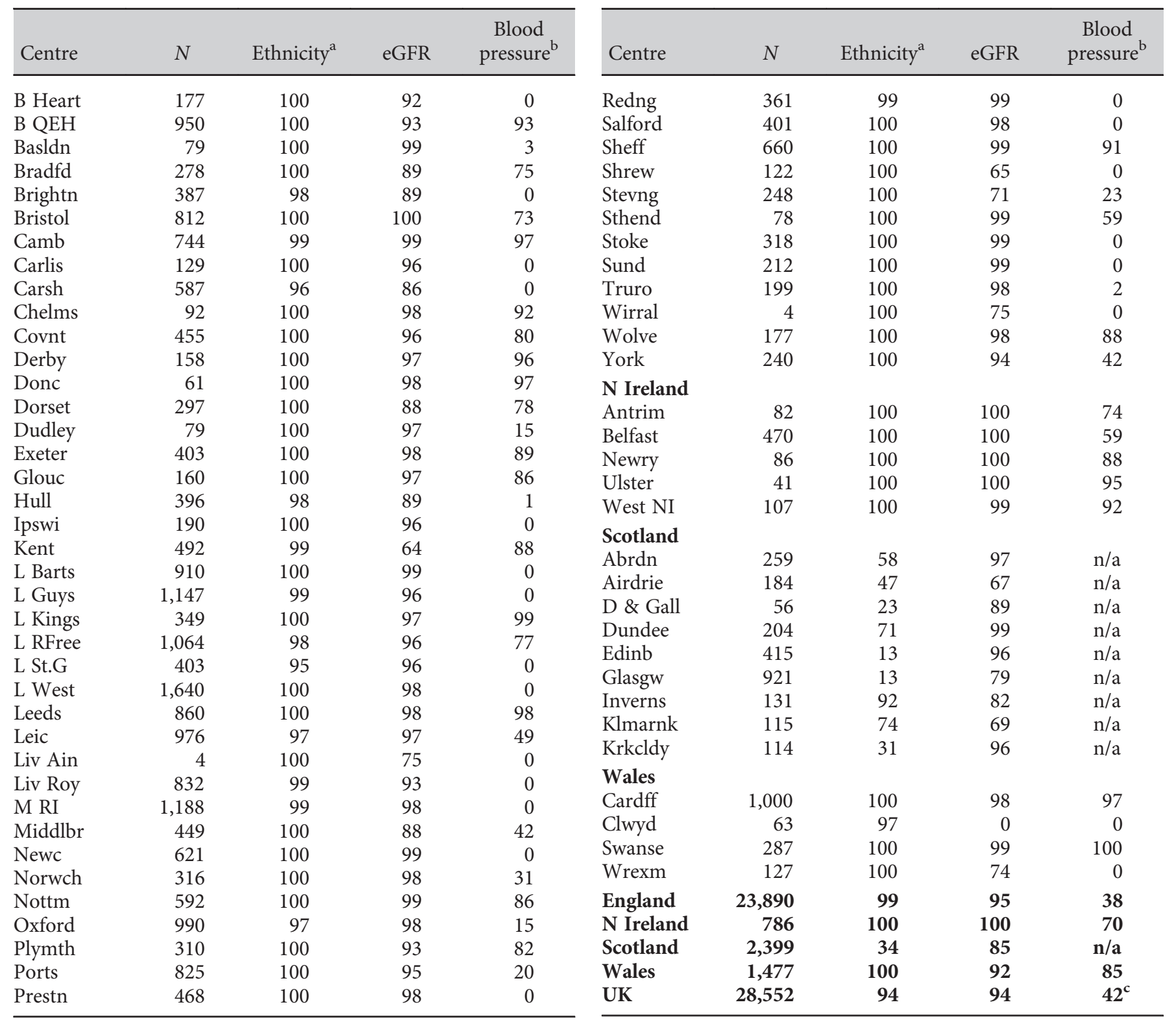

${ }^{\text {a }}$ Patients with missing ethnicity were classed as White for eGFR calculation

${ }^{\mathrm{b}} \mathrm{S}$ cottish centres excluded from blood pressure analysis as data not provided by the Scottish Renal Registry

${ }^{\mathrm{c}}$ Excluding Scotland

Time since transplantation may have a significant effect on key biochemical and clinical variables and this is likely to be independent of a centre's clinical practices. Therefore, inter-centre comparison of data on prevalent transplant patients is open to bias. To minimise bias relating to fluctuations in biochemical and clinical parameters occurring in the initial post-transplant period, one year post-transplantation outcomes are also reported. It is presumed that patient selection policies and local clinical practices are more likely to be relevant in influencing outcomes 12 months post-transplant and therefore comparison of outcomes between centres is more robust. However, even the 12 months post-transplant comparisons could be biased by the fact that in some centres, repatriation of patients only occurs if the graft is failing whereas in others it only occurs if the graft function is stable.

Centres with $<20$ patients or $<50 \%$ data completeness have been excluded from the figures. Scottish centres were also excluded from blood pressure analyses as data were not provided.

\section{Prevalent patient data}

Biochemical and clinical data for patients with a functioning transplant followed in either a transplanting or non-transplanting centre were included in the analyses. The cohort consisted of 
Table 3.9b. Percentage completeness of haemoglobin, serum cholesterol, serum calcium, serum phosphate and serum PTH by centre for prevalent transplant patients on $31 / 12 / 2013$

\begin{tabular}{|c|c|c|c|c|c|c|}
\hline Centre & $N$ & Haemoglobin & $\begin{array}{l}\text { Total serum } \\
\text { cholesterol }^{\mathrm{a}}\end{array}$ & $\begin{array}{l}\text { Adjusted serum } \\
\text { calcium }^{\mathrm{a}, \mathrm{b}}\end{array}$ & $\begin{array}{c}\text { Serum } \\
\text { phosphate }\end{array}$ & $\begin{array}{l}\text { Serum } \\
\text { PTH }^{\mathrm{a}}\end{array}$ \\
\hline \multicolumn{7}{|l|}{ England } \\
\hline B QEH & 950 & 92 & 90 & 93 & 92 & 72 \\
\hline Basldn & 79 & 99 & 49 & 99 & 94 & 44 \\
\hline Bradfd & 278 & 87 & 73 & 78 & 62 & 46 \\
\hline Camb & 744 & 98 & 97 & 99 & 99 & 97 \\
\hline Carlis & 129 & 95 & 61 & 95 & 91 & 9 \\
\hline Carsh & 587 & 80 & 60 & 86 & 86 & 19 \\
\hline Chelms & 92 & 96 & 89 & 98 & 93 & 26 \\
\hline Covnt & 455 & 96 & 0 & 95 & 70 & 40 \\
\hline Exeter & 403 & 98 & 86 & 97 & 97 & 25 \\
\hline Glouc & 160 & 96 & 66 & 96 & 96 & 24 \\
\hline Hull & 396 & 89 & 27 & 89 & 88 & 7 \\
\hline Ipswi & 190 & 95 & 57 & 96 & 96 & 67 \\
\hline Kent & 492 & 97 & 77 & 95 & 95 & 17 \\
\hline L Barts & 910 & 98 & 99 & 99 & 99 & 92 \\
\hline L Guys & 1,147 & 0 & 55 & 90 & 90 & 40 \\
\hline L Kings & 349 & 97 & 79 & 97 & 97 & 32 \\
\hline L RFree & 1,064 & 95 & 81 & 95 & 95 & 77 \\
\hline L St.G & 403 & 96 & 81 & 95 & 95 & 84 \\
\hline L West & 1,640 & 98 & 45 & 98 & 98 & 36 \\
\hline Nottm & 592 & 98 & 84 & 96 & 93 & 88 \\
\hline Oxford & 990 & 98 & 74 & 98 & 98 & 34 \\
\hline Plymth & 310 & 92 & 56 & 88 & 87 & 39 \\
\hline Ports & 825 & 95 & 57 & 93 & 88 & 24 \\
\hline Prestn & 468 & 97 & 71 & 95 & 94 & 58 \\
\hline Redng & 361 & 99 & 92 & 98 & 85 & 48 \\
\hline Salford & 401 & 98 & 87 & 97 & 97 & 80 \\
\hline Sheff & 660 & 99 & 65 & 98 & 98 & 26 \\
\hline Shrew & 122 & 81 & 79 & 72 & 72 & 14 \\
\hline Stevng & 248 & 96 & 81 & 92 & 80 & 57 \\
\hline Sthend & 78 & 99 & 35 & 99 & 96 & 8 \\
\hline Stoke & 318 & 99 & 100 & 99 & 98 & 69 \\
\hline Sund & 212 & 98 & 96 & 98 & 97 & 95 \\
\hline Truro & 199 & 97 & 61 & 97 & 97 & 34 \\
\hline Wirral & 4 & 75 & 75 & 75 & 75 & 75 \\
\hline Wolve & 177 & 96 & 86 & 93 & 83 & 64 \\
\hline York & 240 & 94 & 64 & 89 & 86 & 20 \\
\hline
\end{tabular}


Table 3.9b. Continued

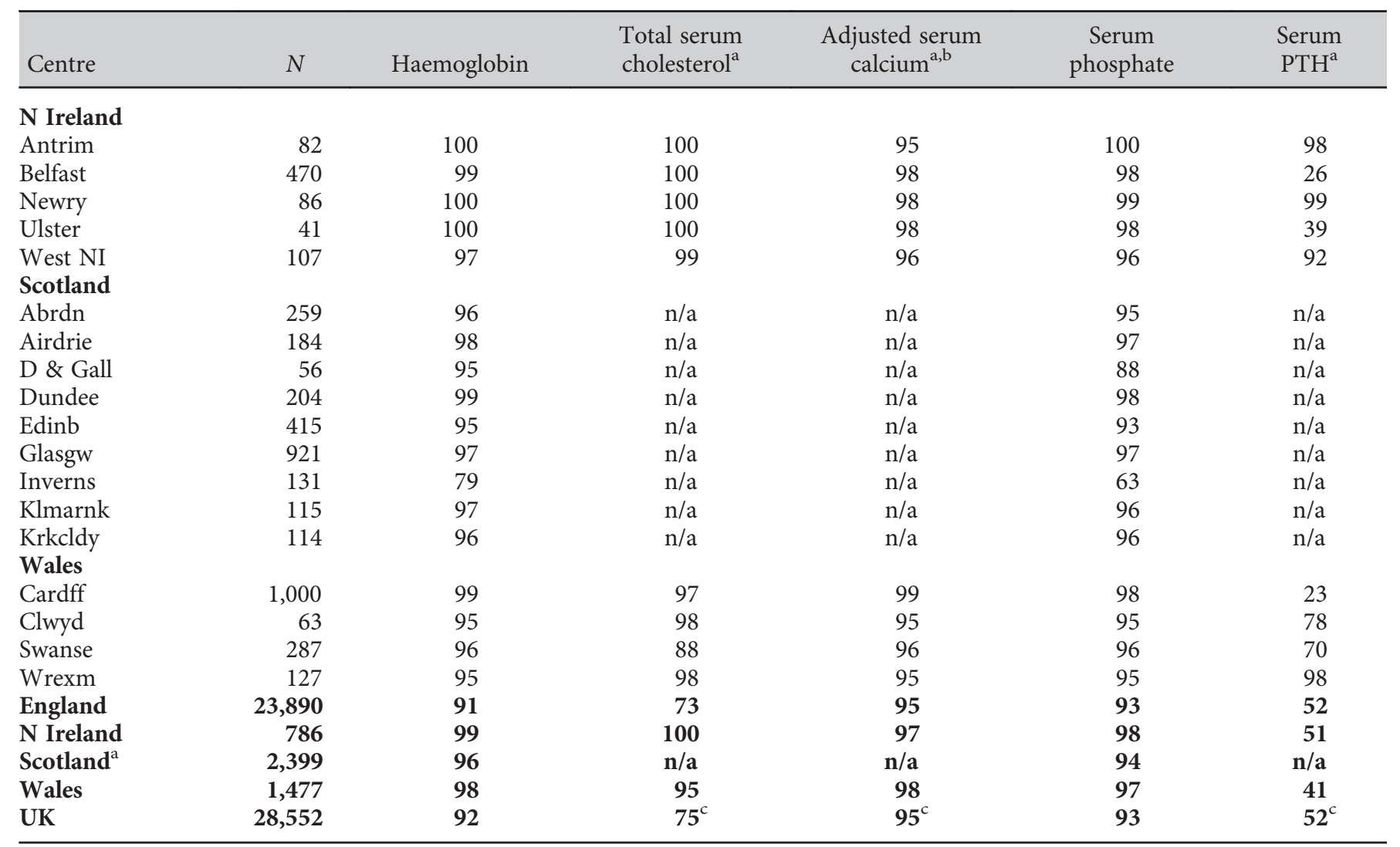

${ }^{a}$ Dataset provided by the Scottish Renal Registry for Scottish centres shown did not include data on serum cholesterol, serum calcium or serum PTH

${ }^{\mathrm{b}}$ Serum calcium corrected for serum albumin

${ }^{\mathrm{c}}$ Excluding Scotland

prevalent patients as on 31st December 2013. Patients were considered as having a functioning transplant if 'transplant' was listed as the last mode of RRT in the last quarter of 2013. Patients were assigned to the renal centre that sent the data to the UKRR but some patients will have received care in more than one centre. If data for the same transplant patient were received from both the transplant centre and non-transplant centre, care was usually allocated to the non-transplant centre (see appendix B2). Patients with a functioning transplant of less than three months duration were excluded from analyses. For haemoglobin, estimated glomerular filtration rate (eGFR), corrected calcium, phosphate and blood pressure (BP), the latest value in quarter 3 or quarter 4 of 2013 was used.

\section{Estimated glomerular filtration rate (eGFR)}

For the purpose of eGFR calculation, the original 4-variable MDRD formula was used (with a constant of 186) to calculate eGFR from the serum creatinine concentration as reported by the centre (unless otherwise stated). A wide variety of creatinine assays are in use in clinical biochemistry laboratories in the UK, and it is not possible to ensure that all measurements of creatinine concentration collected by the UKRR are harmonised. Although many laboratories are now reporting assay results that have been aligned to the isotope dilution-mass spectrometry standard (which would necessitate use of the modified MDRD formula), this was not the case at the end of 2013. Patients with valid serum creatinine results but no ethnicity data were classed as White for the purpose of the eGFR calculation.

\section{One year post-transplant data}

Patients who received a renal transplant between 1st January 2006 and 31st December 2012 were assigned according to the renal centre in which they were transplanted. In a small number of instances, the first documented evidence of transplantation in a patient's record is from a timeline entry in data returned from a non-transplant centre, in these instances the patient was reassigned to the nearest transplant centre (table 3.10).

Patients who had died or experienced graft failure within 12 months of transplantation were excluded from the analyses. Patients with more than one transplant during 2006-2012 were included as separate episodes provided each of the transplants functioned for a year.

For each patient, the most recent laboratory or blood pressure result for the relevant 4 th/5th quarter after renal transplantation was taken to be representative of the one year post-transplant outcome. Again, for the purpose of the eGFR calculation patients with valid serum creatinine results but missing ethnicity data were classed as White. 
Table 3.10. Number of patients per transplant centre after allocation of patients at non-transplant centres (transplanted between 2006-2012)

\begin{tabular}{|c|c|c|c|}
\hline Transplant centre & $\begin{array}{l}\text { Total number of patients } \\
\text { per transplant centre }\end{array}$ & Non-transplant centre & $\begin{array}{c}\text { Number of patients rellocated } \\
\text { to a transplant centre }\end{array}$ \\
\hline $\mathrm{B} \mathrm{QEH}$ & 899 & Stoke & 2 \\
\hline \multirow[t]{4}{*}{ Belfast } & 381 & Antrim & 2 \\
\hline & & Newry & 4 \\
\hline & & Ulster & 3 \\
\hline & & West NI & 4 \\
\hline Bristol & 678 & Dorset & 3 \\
\hline Camb & 1,115 & & \\
\hline Cardff & 790 & Swansea & 2 \\
\hline Covnt & 369 & & \\
\hline \multirow[t]{3}{*}{ Edinb } & 644 & Abrdn & 1 \\
\hline & & Dundee & 6 \\
\hline & & Inverns & 2 \\
\hline Glasgw & 635 & Airdrie & 2 \\
\hline L Barts & 731 & & \\
\hline L Guys & 1,276 & L Kings & 3 \\
\hline L Rfree & 647 & & \\
\hline \multirow[t]{2}{*}{ L St.G } & 581 & Brightn & 1 \\
\hline & & Carsh & 1 \\
\hline L West & 1,129 & & \\
\hline Leeds & 949 & & \\
\hline Leic & 540 & & \\
\hline Liv Roy & 594 & Prestn & 1 \\
\hline M RI & 1,087 & & \\
\hline Newc & 809 & Middlbr & 1 \\
\hline Nottm & 414 & & \\
\hline Oxford & 1,151 & & \\
\hline Plymth & 421 & & \\
\hline Ports & 438 & & \\
\hline Sheff & 410 & & \\
\hline Total & 16,688 & & 38 \\
\hline
\end{tabular}

\section{Results and conclusions}

Post-transplant eGFR in prevalent transplant patients

When interpreting eGFR post-transplantation, it is important to remember that estimated GFR formulae only have a modest predictive performance in the transplant population [4]. Median eGFR in each centre and percentage of patients with eGFR $<30 \mathrm{ml} / \mathrm{min} /$ $1.73 \mathrm{~m}^{2}$ are shown in figures 3.2 and 3.3. The median eGFR was $51.8 \mathrm{ml} / \mathrm{min} / 1.73 \mathrm{~m}^{2}$, with $13.4 \%$ of prevalent transplant recipients having an eGFR $<30 \mathrm{ml} / \mathrm{min} /$ $1.73 \mathrm{~m}^{2}$. Table 3.11 summarises the proportion of transplant patients with an eGFR $<30 \mathrm{ml} / \mathrm{min} / 1.73 \mathrm{~m}^{2}$ by centre. Whilst local repatriation policies on timing of transfer of care for patients with failing transplants from transplant centres to referring centres might explain some of the differences, it is notable that both transplanting and non-transplanting centres feature at both ends of the scale. The accuracy of the 4 -variable MDRD equation in estimating GFR $\geqslant 60 \mathrm{ml} / \mathrm{min} / 1.73 \mathrm{~m}^{2}$ is questionable [5], therefore a figure describing this is not included in this chapter.

Figure 3.4 shows the percentage of prevalent patients by centre with eGFR $<30 \mathrm{ml} / \mathrm{min} / 1.73 \mathrm{~m}^{2}$ as a funnel plot, enabling a more reliable comparison of outcomes between centres across the UK. The solid lines show the 2 standard deviation limits (95\%) and the dotted lines the limits for 3 standard deviations (99.9\%). With 66 centres included and a normal distribution, 3-4 centres would be expected to fall between the $95-99.9 \%$ CI (1 in 20) and no centres should fall outside the $99.9 \%$ limits.

There continued to be variation between centres; these data show over-dispersion with 16 centres falling outside the $95 \%$ CI of which six centres were outside the $99.9 \%$ CI. Three centres (Newry, London St Georges, London 


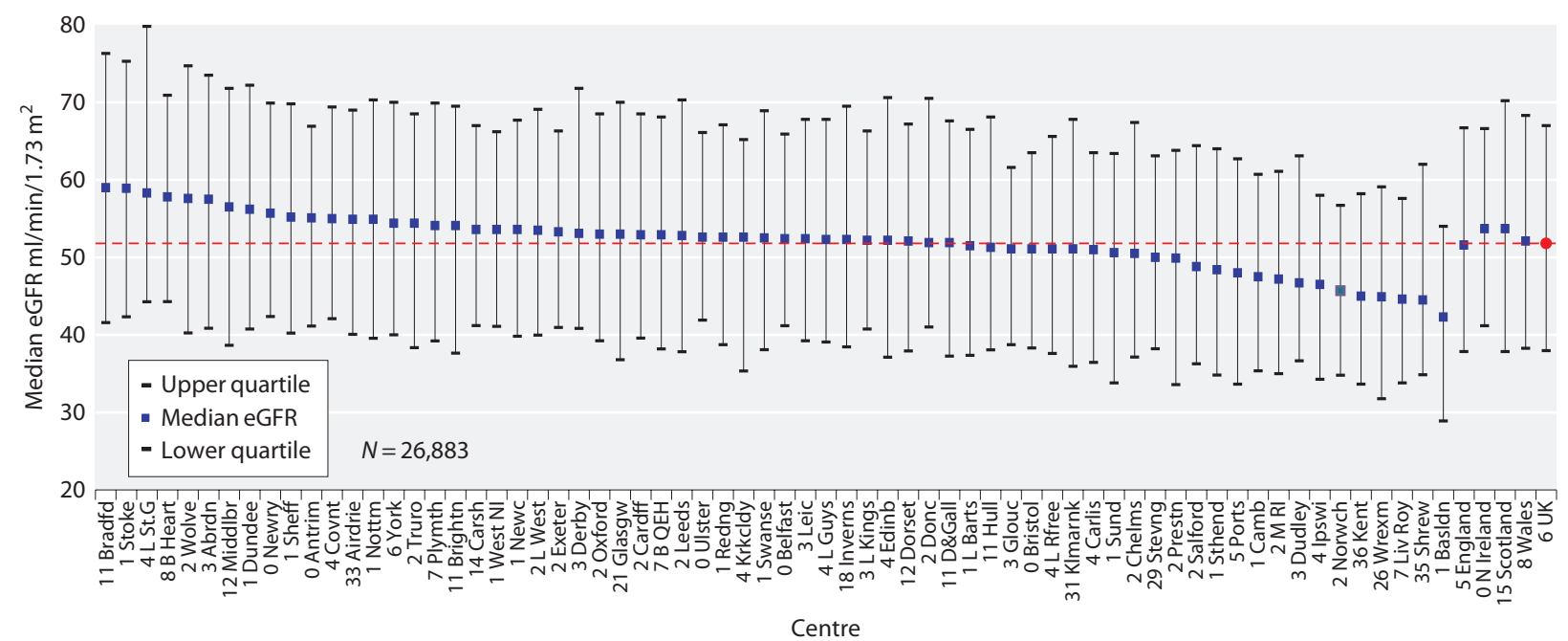

Fig. 3.2. Median eGFR in prevalent transplant patients by centre on $31 / 12 / 2013$

West) fell outside the lower 99.9\% CI suggesting a lower than expected proportion of patients with eGFR $<30 \mathrm{ml} /$ $\mathrm{min} / 1.73 \mathrm{~m}^{2}$. Liverpool Royal, Portsmouth and Preston fell outside the upper $99.9 \%$ CI suggesting a higher than expected proportion of patients with eGFR $<30 \mathrm{ml} / \mathrm{min} / 1.73 \mathrm{~m}^{2}$.

eGFR in patients one year after transplantation

Graft function at one year post-transplantation may predict subsequent long term graft outcome [6]. Figures $3.5 \mathrm{a}, 3.5 \mathrm{~b}$, and $3.5 \mathrm{c}$ show the median one year post-transplant eGFR for patients transplanted between 2006-2012, by transplant type. Living kidney donation had the highest median eGFR at one year $(56.9 \mathrm{ml} / \mathrm{min} /$
$1.73 \mathrm{~m}^{2}$ ), followed by donation after brainstem death $\left(53 \mathrm{ml} / \mathrm{min} / 1.73 \mathrm{~m}^{2}\right)$ and donation after circulatory death $\left(49.7 \mathrm{ml} / \mathrm{min} / 1.73 \mathrm{~m}^{2}\right)$.

Figures 3.6a, 3.6b and 3.6c show one year posttransplant eGFR by donor type and year of transplantation. An upward trend in eGFR $(p=0.001)$ over the time period was noticed with both live and donation after brainstem death transplant, but not with donation after circulatory death $(p=0.4)$.

\section{Haemoglobin in prevalent transplant patients}

Transplant patients have previously fallen under the remit of the UK Renal Association Complications of Chronic Kidney Disease (CKD) guidelines. Updated

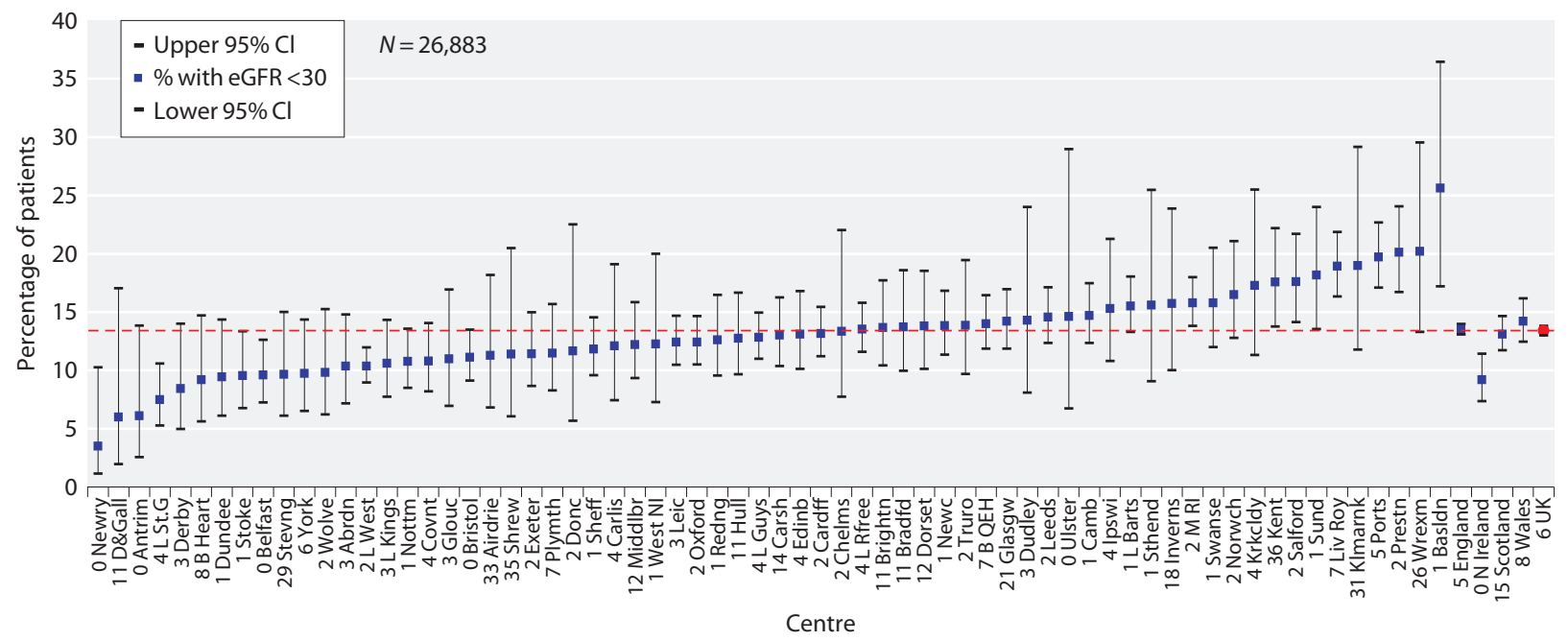

Fig. 3.3. Percentage of prevalent transplant patients by centre on $31 / 12 / 2013$ with eGFR $<30 \mathrm{ml} / \mathrm{min} / 1.73 \mathrm{~m}{ }^{2}$ 
Table 3.11. Percentage of prevalent transplant patients with eGFR $<30 \mathrm{ml} / \mathrm{min} / 1.73 \mathrm{~m}^{2}$ on $31 / 12 / 2013$

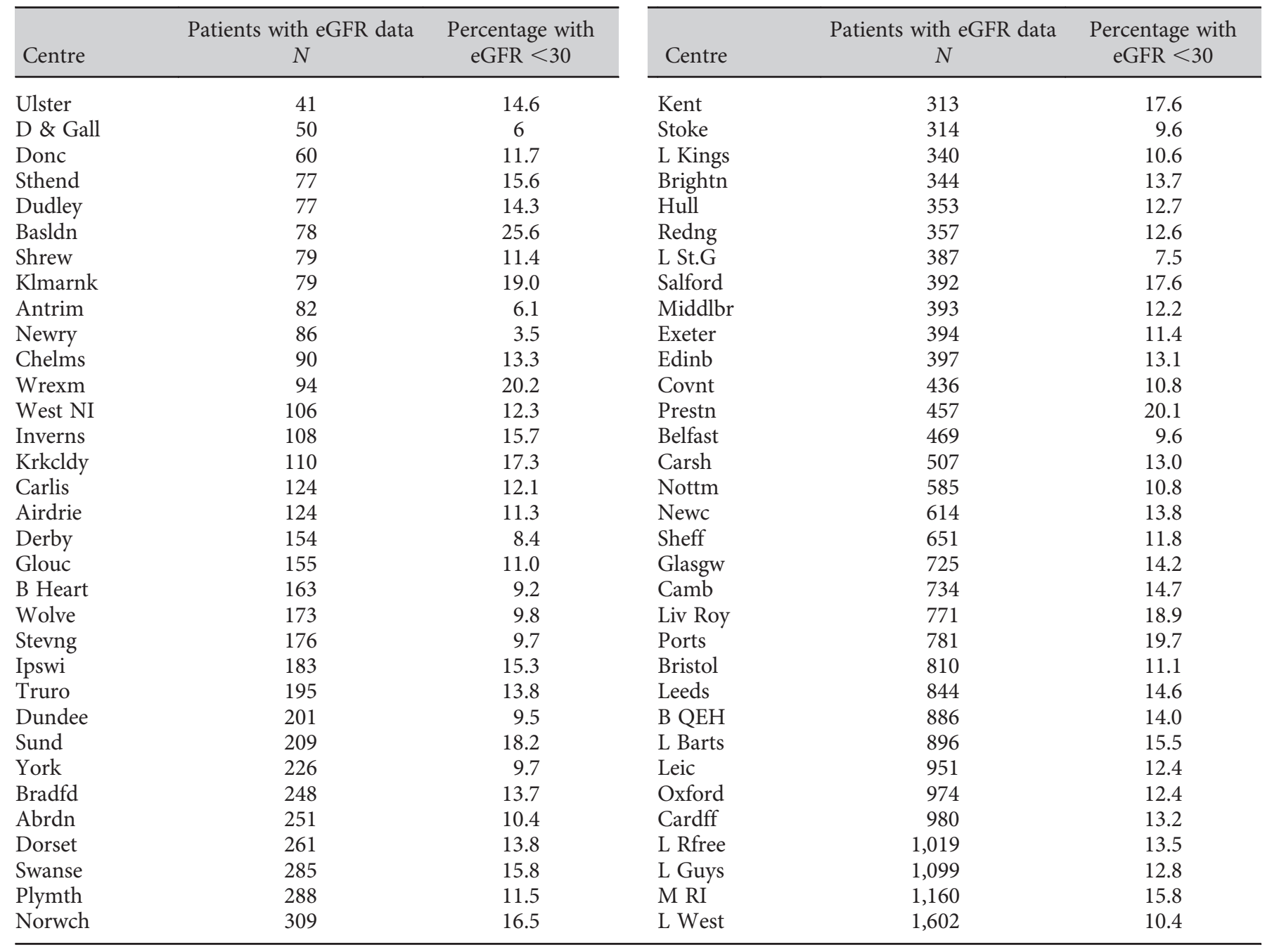

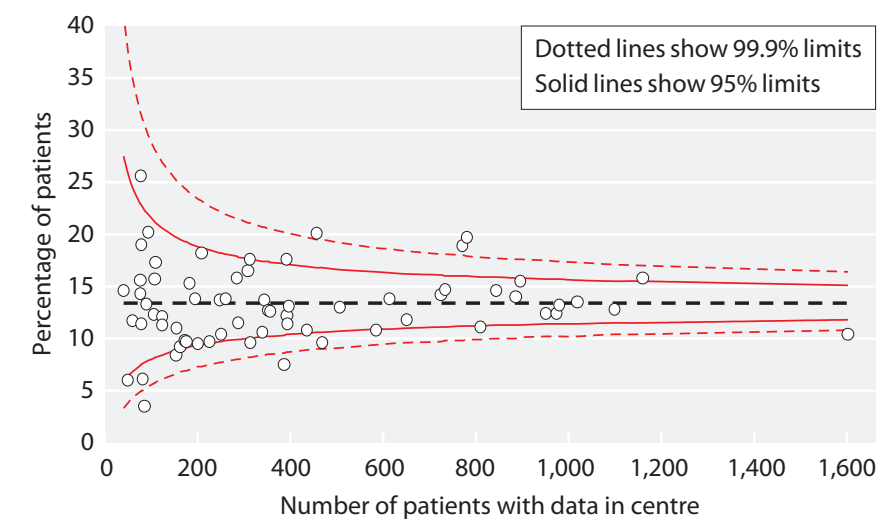

Fig. 3.4. Funnel plot of percentage of prevalent transplant patients with eGFR $<30 \mathrm{ml} / \mathrm{min} / 1.73 \mathrm{~m}^{2}$ by centre size on $31 /$ $12 / 2013$ guidelines regarding the management of anaemia in CKD were published by the association in November 2010 [7] which have now been adopted for this report. These guidelines recommend 'achieving a population distribution centred on a mean of $11 \mathrm{~g} / \mathrm{dl}$ with a range of 10-12 $\mathrm{g} / \mathrm{dl}$ ' [8] (equivalent to $110 \mathrm{~g} / \mathrm{L}$, range 100-120 g/ L). However, many transplant patients with good transplant function will have haemoglobin concentrations $>120 \mathrm{~g} / \mathrm{L}$ without the use of erythopoiesis stimulating agents, and so it is inappropriate to audit performance using the higher limit.

A number of factors including comorbidity, immunosuppressive medication, graft function, ACE inhibitor use, erythropoietin (EPO) use, intravenous or oral iron use, as well as centre practices and protocols for management of anaemia, affect haemoglobin concentrations in transplant patients. Most of these data are not collected 


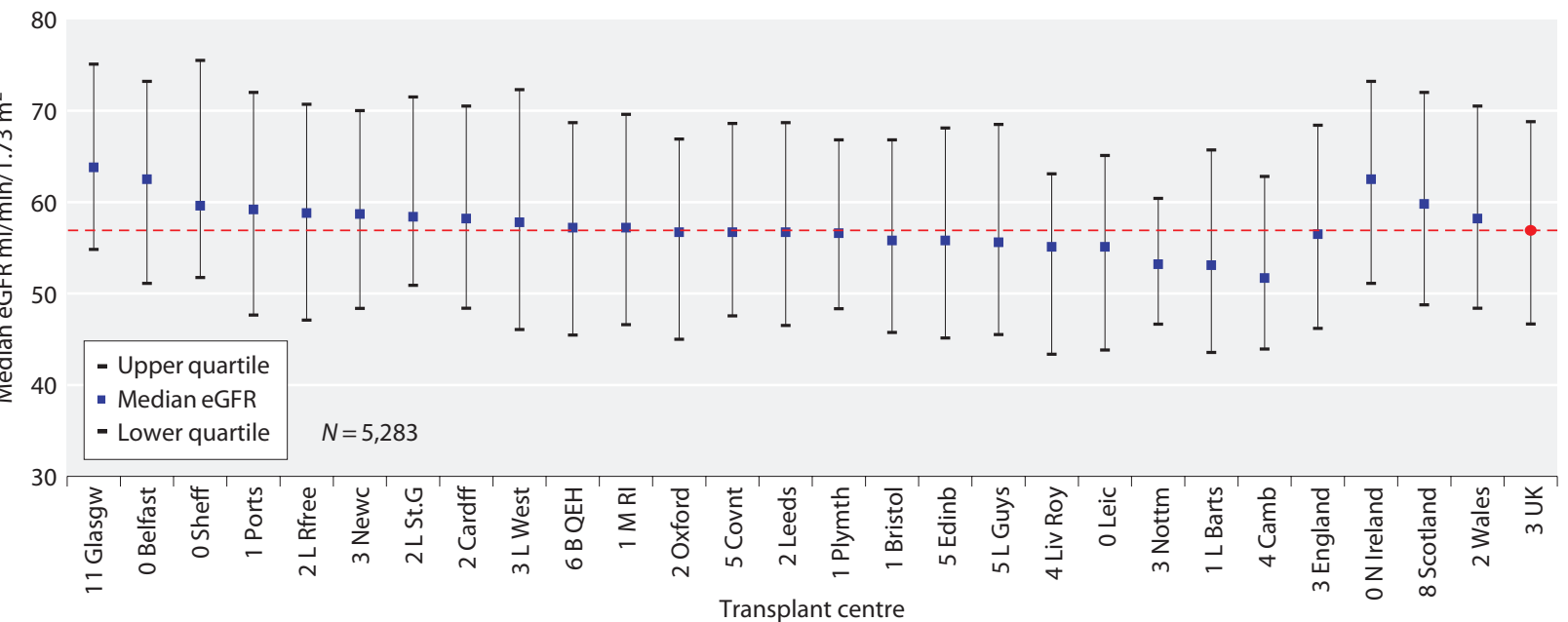

Fig. 3.5a. Median eGFR one year post-live donor transplant by transplant centre 2006-2012

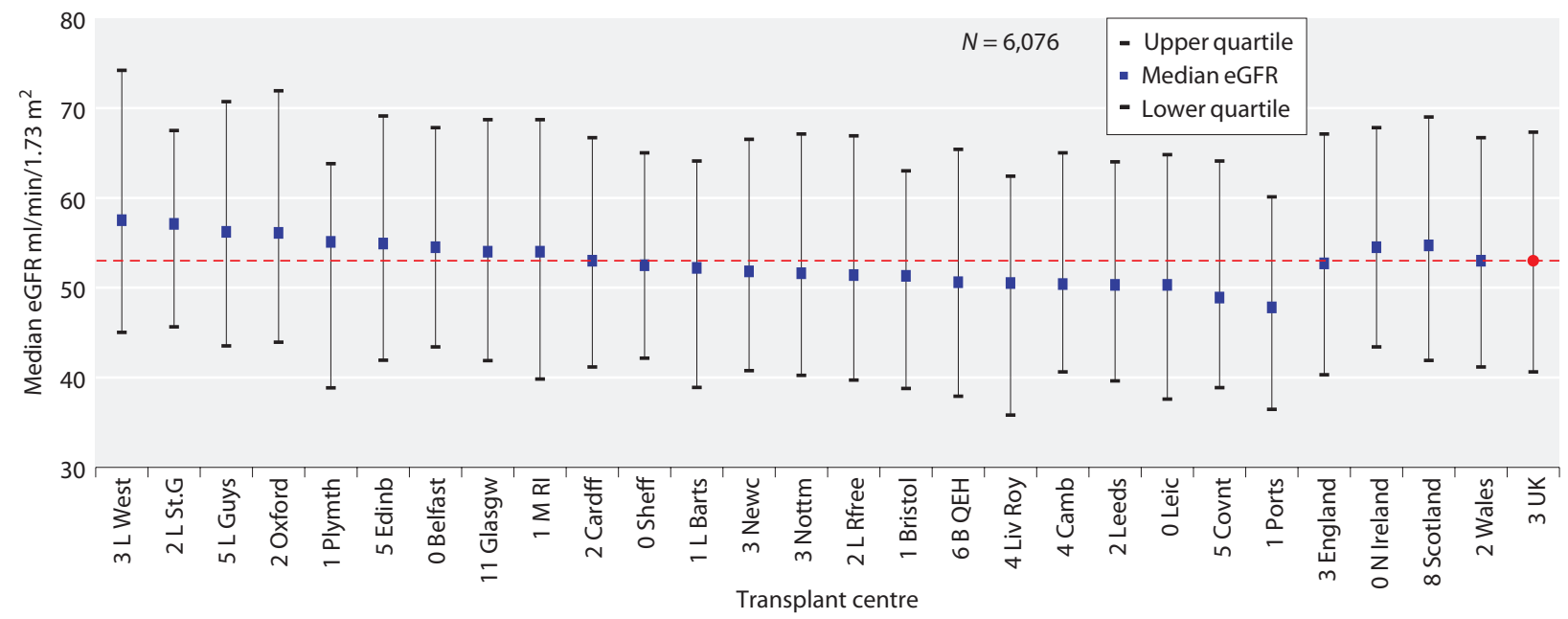

Fig. 3.5b. Median eGFR one year post-brainstem death donor transplant by transplant centre 2006-2012

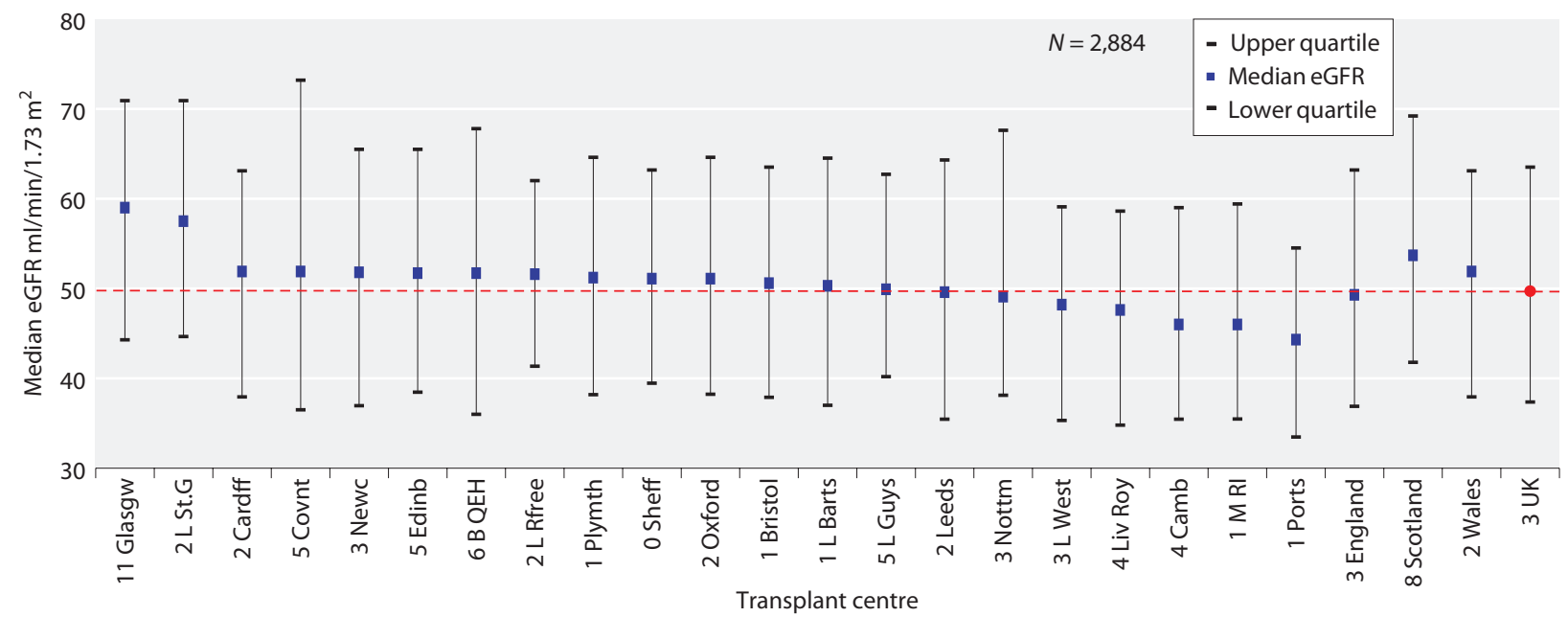

Fig. 3.5c. Median eGFR one year post-circulatory death donor transplant by transplant centre 2006-2012 


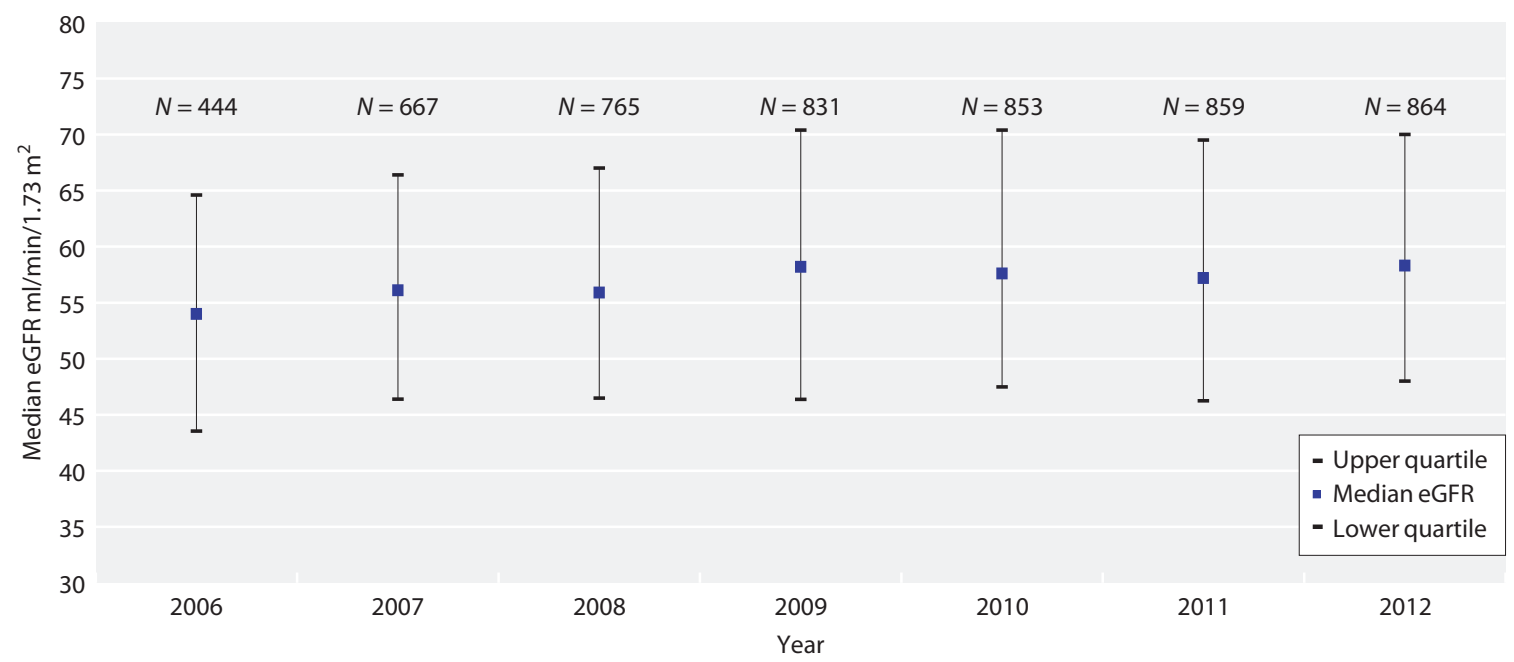

Fig. 3.6a. Median eGFR one year post-live donor transplant by year of transplantation 2006-2012

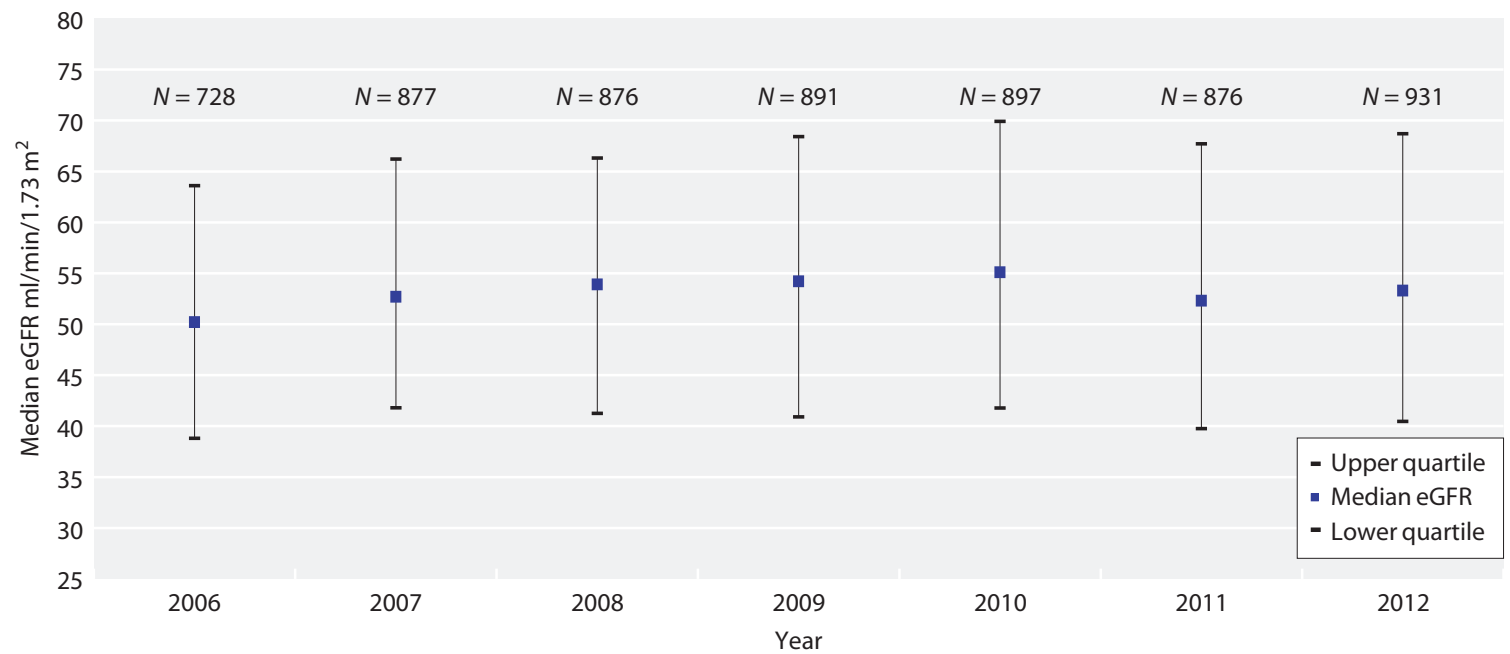

Fig. 3.6b. Median eGFR one year post-brainstem death donor transplant by year of transplantation 2006-2012

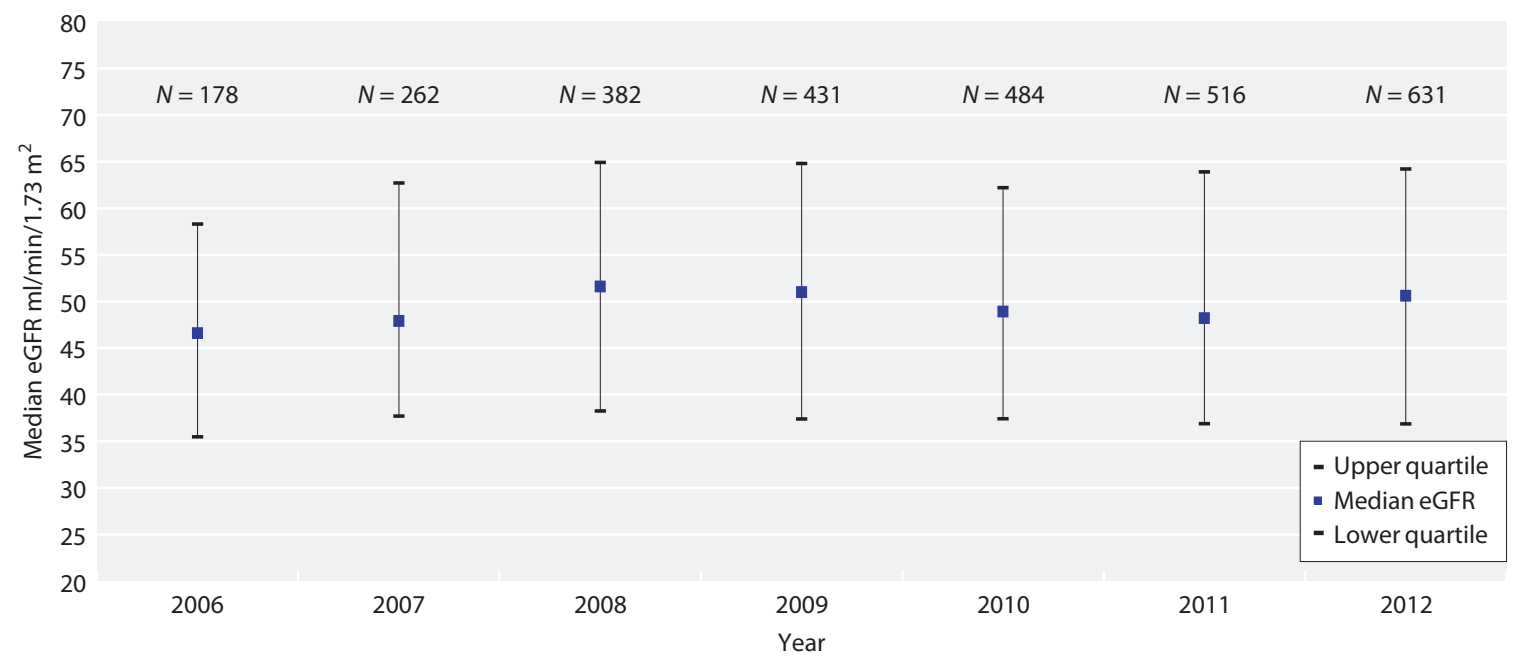

Fig. 3.6c. Median eGFR one year post-circulatory death donor transplant by year of transplantation 2006-2012 
by the UKRR and therefore caution must be used when interpreting analyses of haemoglobin attainment. Figures $3.7 \mathrm{a}$ and $3.7 \mathrm{~b}$ report centre results stratified according to graft function as estimated by eGFR. The percentage of prevalent transplant patients achieving $\mathrm{Hb} \geqslant 100 \mathrm{~g} / \mathrm{L}$ in each centre, stratified by eGFR, is displayed in figures $3.8 \mathrm{a}$ and $3.8 \mathrm{~b}$.

Figure 3.9 describes the percentage of prevalent patients by centre with haemoglobin $<100 \mathrm{~g} / \mathrm{L}$ as a funnel plot enabling more reliable comparison of outcomes between centres across the UK. With 66 centres included and a normal distribution, 3-4 centres would be expected to fall between the $95 \%-99.9 \%$ CI ( 1 in 20 ) and no centres should fall outside the $99.9 \%$ CI purely as a chance event.
Two centres (London St Bartholemews and London Royal Free) fell outside the upper 99.9\% CI and two further centres (Leeds and Oxford) fell outside the upper 95\% CI indicating a higher than predicted proportion of transplant patients not achieving the haemoglobin target. Six centres fell outside the lower $99.9 \% \mathrm{CI}$, indicating they performed better than expected with fewer than predicted patients having a haemoglobin $<100 \mathrm{~g} / \mathrm{L}$.

\section{Blood pressure in prevalent transplant patients}

In the absence of controlled trial data, the opinion based recommendation of the UK Renal Association (RA) published in the 2010 guideline for the care of kidney transplant recipients is that 'Blood pressure should be

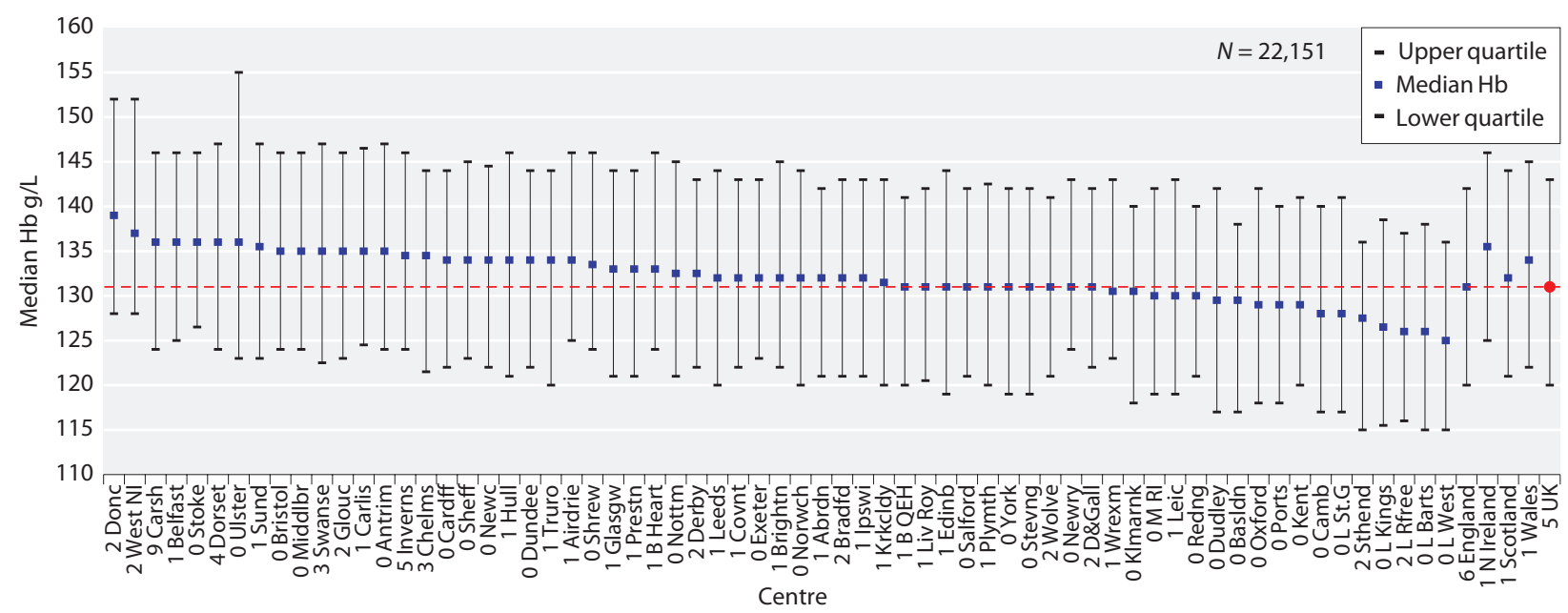

Fig. 3.7a. Median haemoglobin for prevalent transplant patients with eGFR $\geqslant 30 \mathrm{ml} / \mathrm{min} / 1.73 \mathrm{~m}^{2}$ by centre on $31 / 12 / 2013$

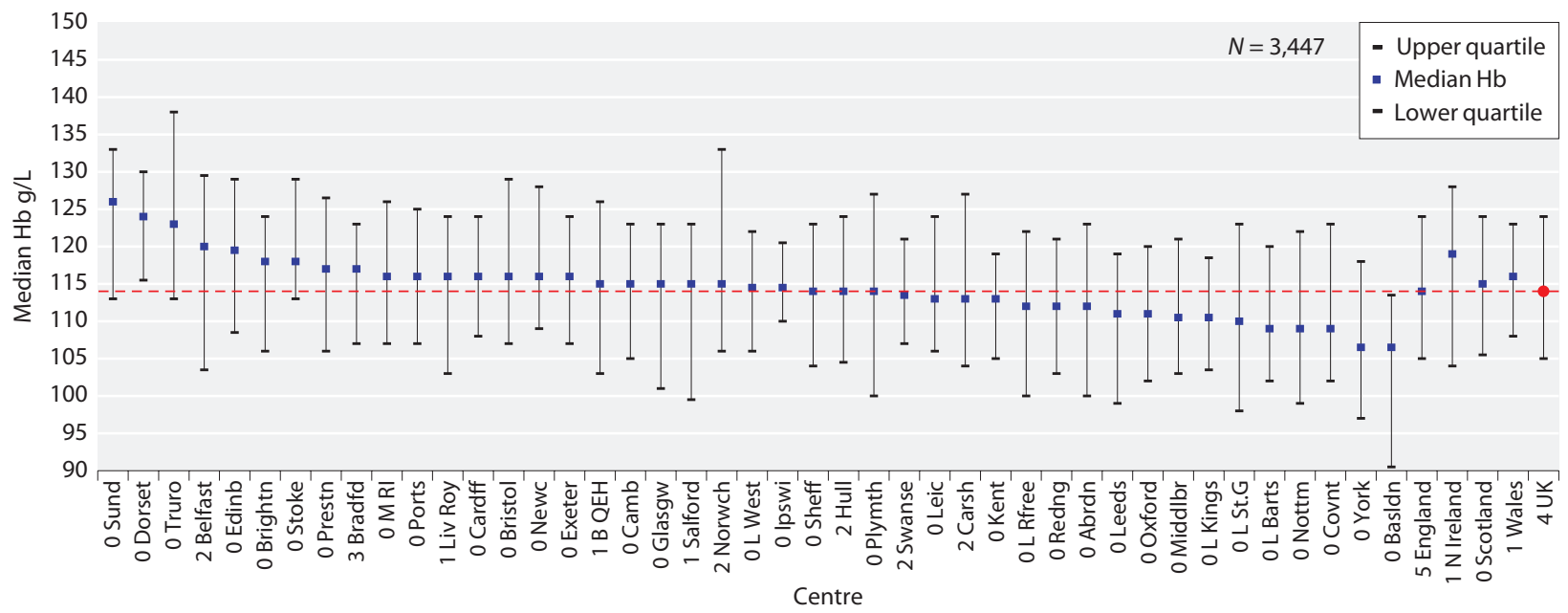

Fig. 3.7b. Median haemoglobin for prevalent transplant patients with eGFR $<30 \mathrm{ml} / \mathrm{min} / 1.73 \mathrm{~m}^{2}$ by centre on $31 / 12 / 2013$ 


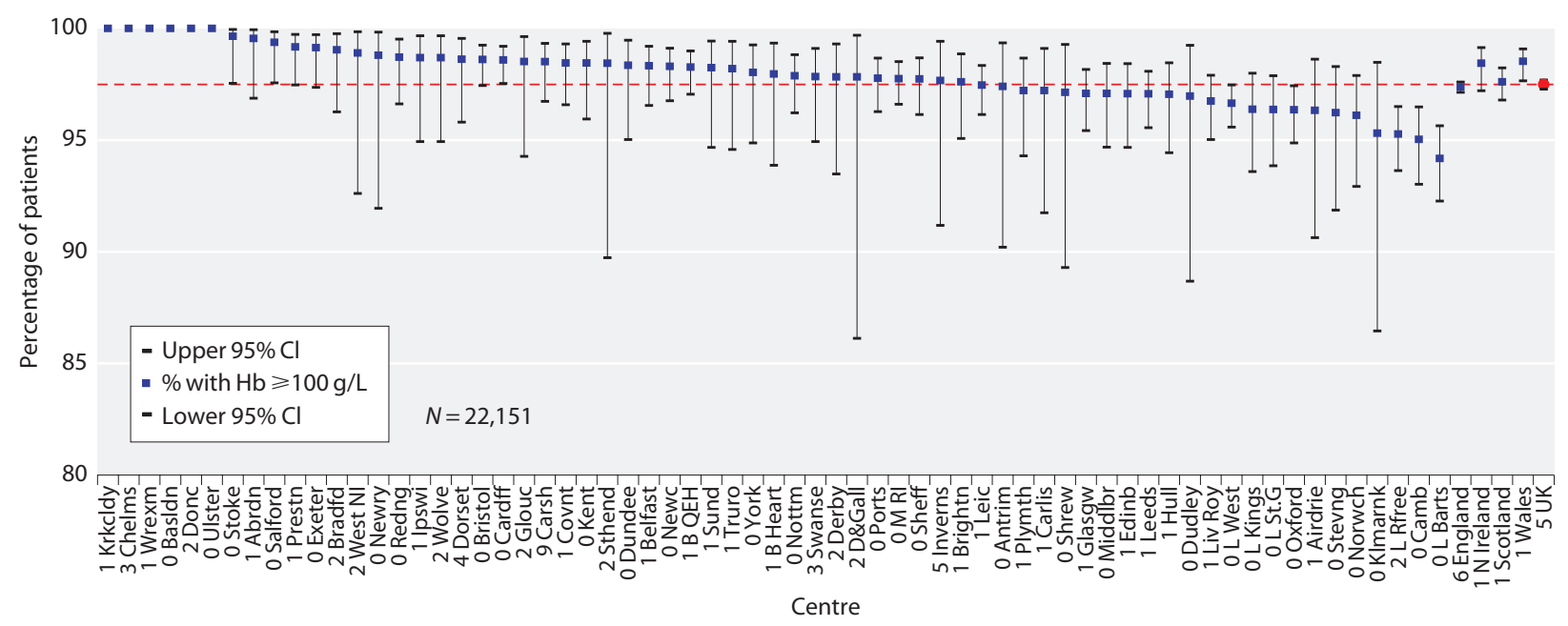

Fig. 3.8a. Percentage of prevalent transplant patients with eGFR $\geqslant 30 \mathrm{ml} / \mathrm{min} / 1.73 \mathrm{~m}^{2}$ achieving haemoglobin $\geqslant 100 \mathrm{~g} / \mathrm{L}$ by centre on $31 / 12 / 2013$

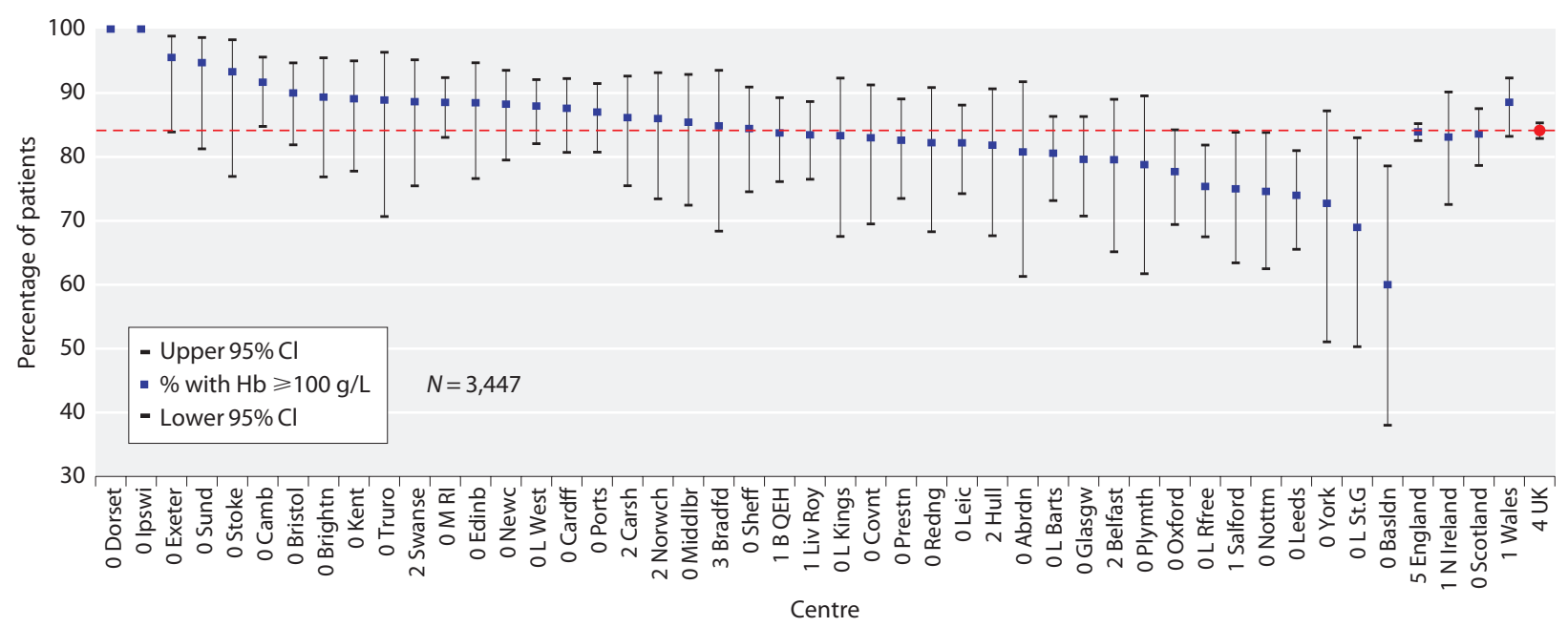

Fig. 3.8b. Percentage of prevalent transplant patients with eGFR $<30 \mathrm{ml} / \mathrm{min} / 1.73 \mathrm{~m}^{2}$ achieving haemoglobin $\geqslant 100 \mathrm{~g} / \mathrm{L}$ by centre on $31 / 12 / 2013$

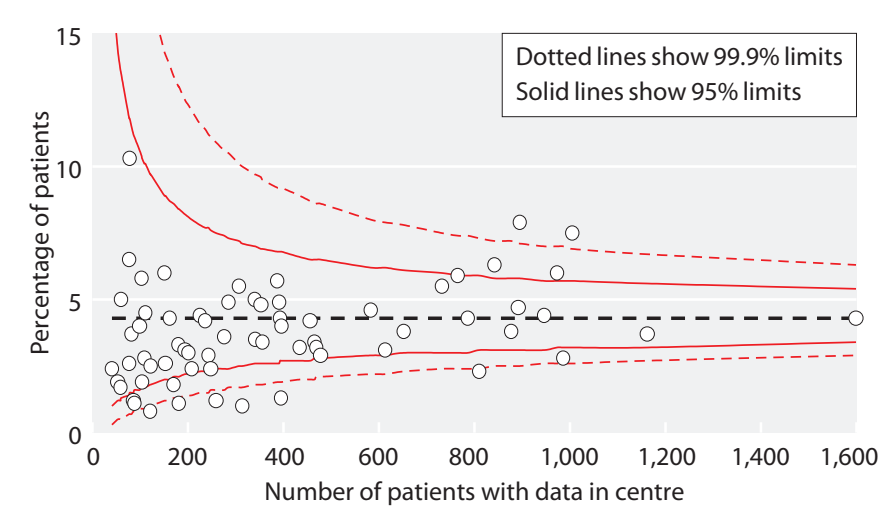

Fig. 3.9. Funnel plot of percentage of prevalent transplant patients with haemoglobin $<100 \mathrm{~g} / \mathrm{L}$ by centre size on $31 / 12 / 2013$
$<130 / 80 \mathrm{mmHg}$ (or $<125 / 75 \mathrm{mmHg}$ if proteinuria)' [9]. This blood pressure target is the same as that used in previous annual reports [10].

As indicated in table 3.9a, completeness for blood pressure data returns was variable and only centres with $>50 \%$ data returns were included for consideration. Despite this restriction, caution needs to be exercised in interpretation of these results because of the volume of missing data and potential bias, (e.g. a centre may be more likely to record and report blood pressure data electronically in patients with poor BP control). Figures $3.10 \mathrm{a}$ and $3.10 \mathrm{~b}$ show the percentage of patients with a blood pressure of $<130 / 80 \mathrm{mmHg}$, by eGFR. The percentage of patients with $\mathrm{BP}<130 / 80$ (systolic 


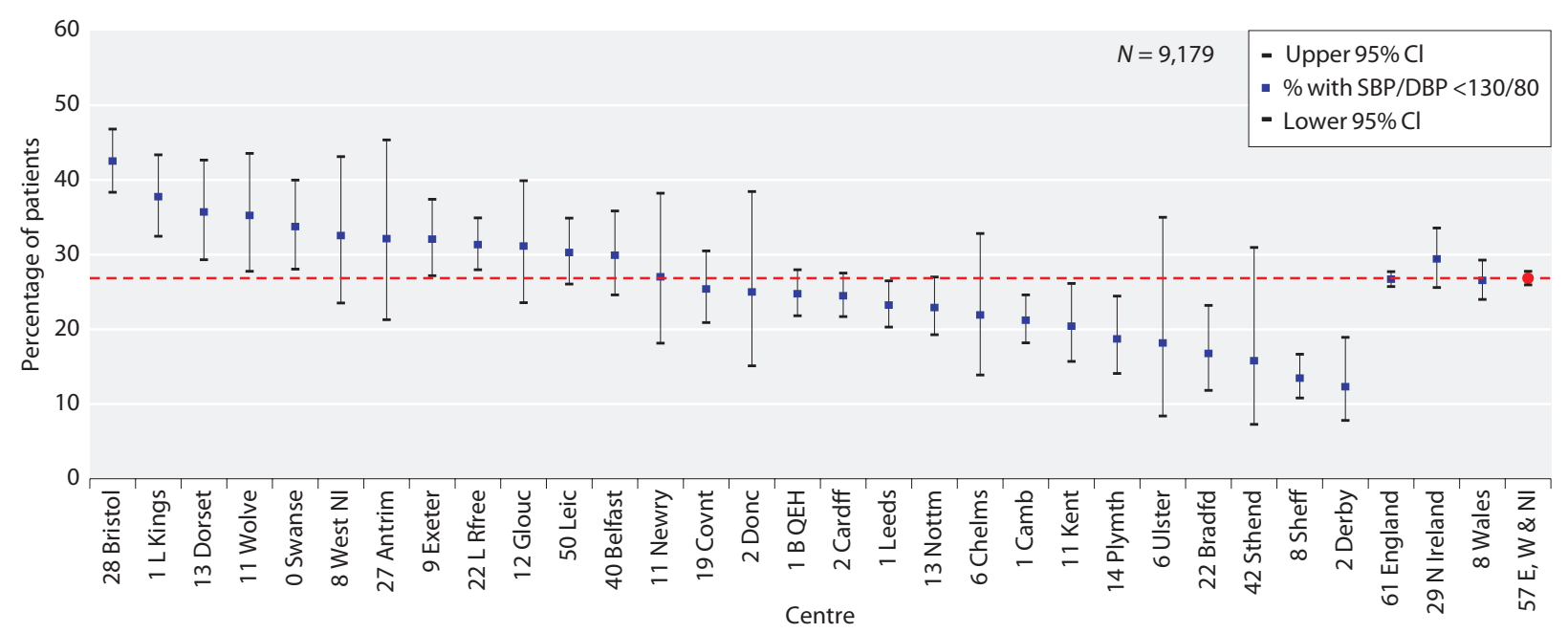

Fig. 3.10a. Percentage of prevalent transplant patients with eGFR $\geqslant 30 \mathrm{ml} / \mathrm{min} / 1.73 \mathrm{~m}^{2}$ achieving blood pressure of $<130 / 80 \mathrm{mmHg}$ by centre on $31 / 12 / 2013$

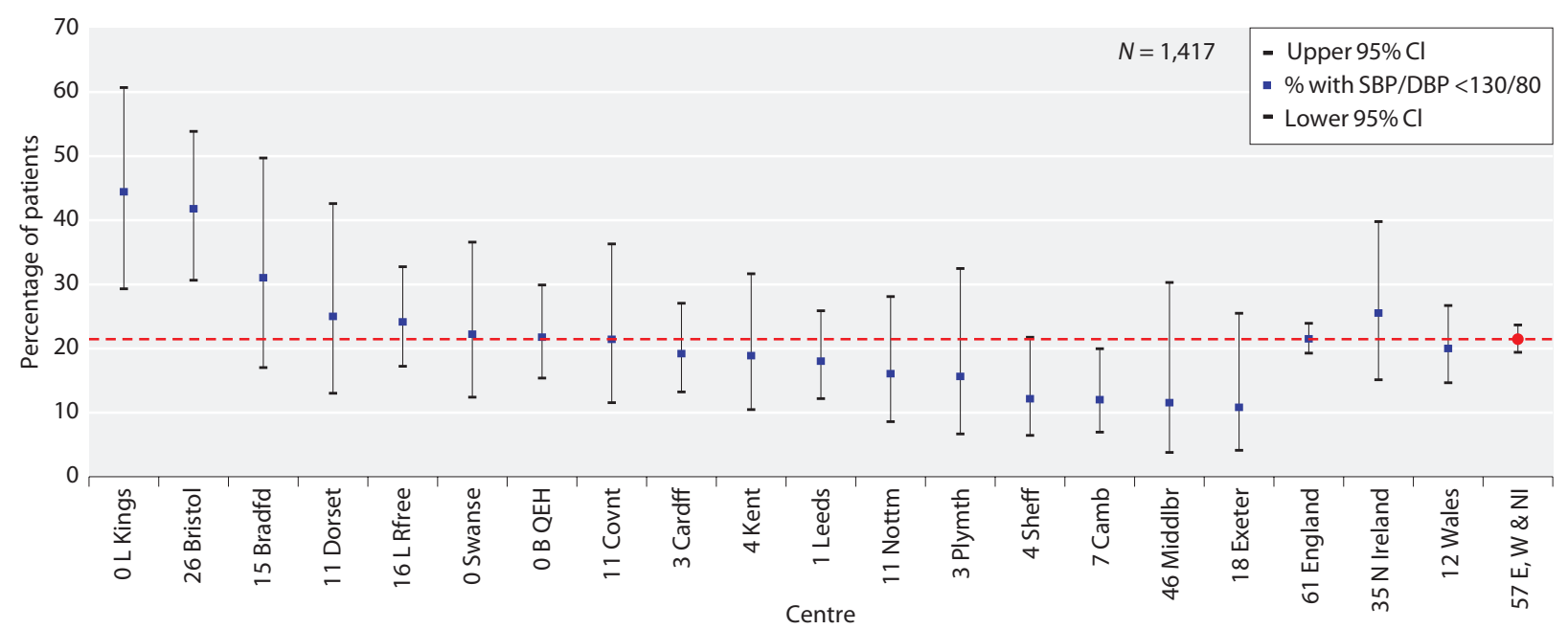

Fig. 3.10b. Percentage of prevalent transplant patients with eGFR $<30 \mathrm{ml} / \mathrm{min} / 1.73 \mathrm{~m}^{2}$ achieving blood pressure of $<130 / 80 \mathrm{mmHg}$ by centre on $31 / 12 / 2013$

$\mathrm{BP}<130$ and diastolic $\mathrm{BP}<80 \mathrm{mmHg}$ ) was higher $(26.8 \%$ vs. $21.5 \%)$ in those with better renal function $\left(\mathrm{eGFR} \geqslant 30 \mathrm{ml} / \mathrm{min} / 1.73 \mathrm{~m}^{2}\right.$ ).

\section{Analysis of prevalent patients by CKD stage}

\section{Introduction}

Approximately $2.4 \%$ of prevalent transplant patients returned to dialysis in 2013, a similar percentage to that seen over the last few years. Amongst patients with native chronic kidney disease, late presentation is associated with poor outcomes, largely attributable to lack of specialist management of anaemia, acidosis, hyperphosphataemia and to inadequate advance preparation for dialysis. Transplant recipients on the other hand, are almost always followed up regularly in specialist transplant or renal clinics and it would be reasonable to expect patients with failing grafts to receive appropriate care and therefore have many of their modifiable risk factors addressed before complete graft failure and return to dialysis.

\section{Methods}

The transplant cohort consisted of prevalent transplant recipients as on 31st December $2013(N=26,896)$ and were classified 
Table 3.12. Analysis by CKD stage for prevalent transplant patients compared with prevalent dialysis patients on $31 / 12 / 2013$

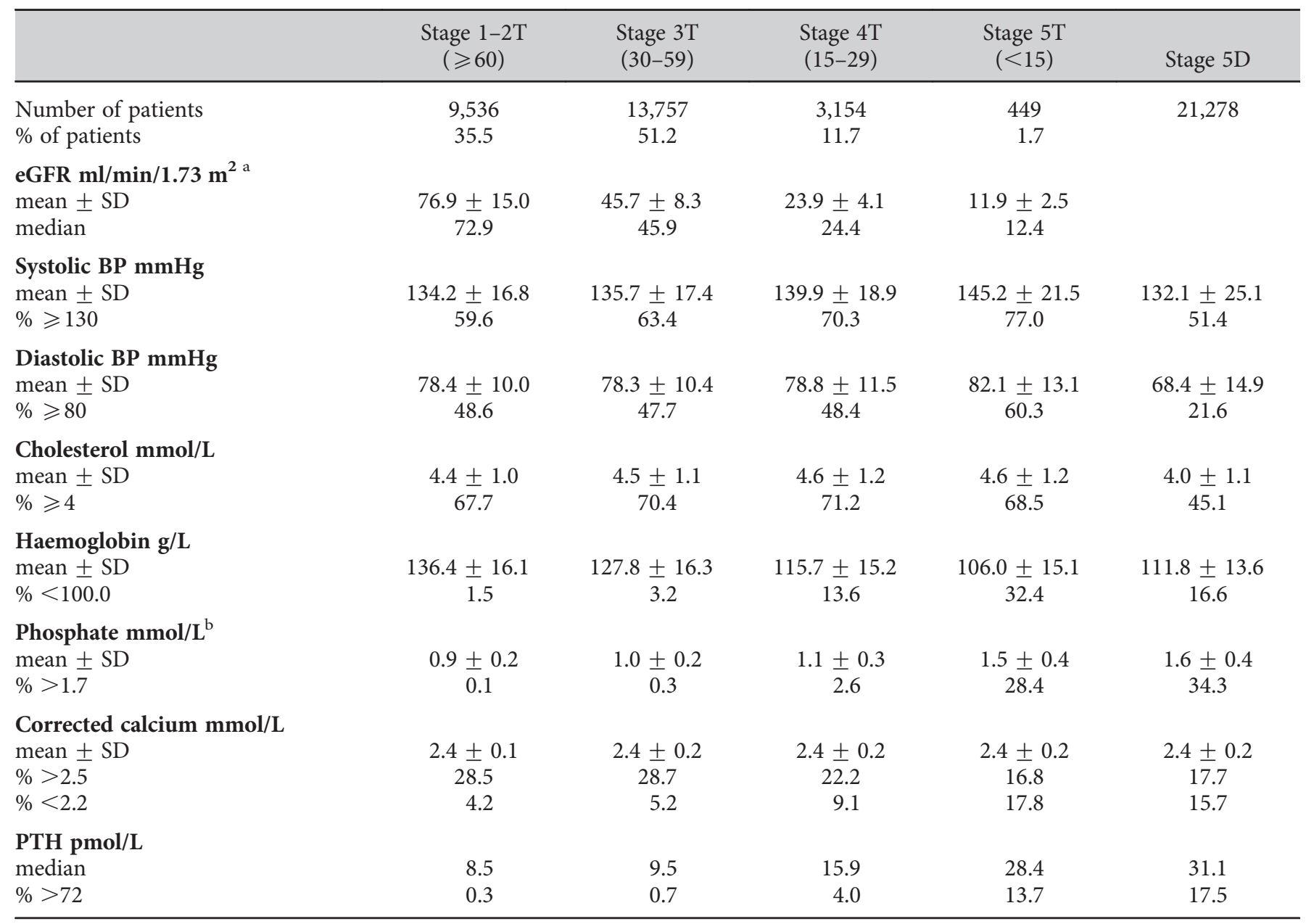

${ }^{\mathrm{a}}$ Prevalent transplant patients with no ethnicity data were classed as White

${ }^{\mathrm{b}}$ Only PD patients included in stage $5 \mathrm{D}, n=2,330$

according to the KDIGO staging criteria with the suffix of ' $\mathrm{T}$ ' to represent their transplant status. Patients with missing ethnicity information were classified as White for the purpose of calculating eGFR. Prevalent dialysis patients, except those who commenced dialysis in 2013, comprised the comparison dialysis cohort $(N=21,278)$ including 2,330 peritoneal dialysis patients. Only patients on peritoneal dialysis were considered when examining differences in serum phosphate between transplant recipients and dialysis patients. For both the transplant and dialysis cohorts, the analysis used the most recent available value from the last two quarters of the 2013 laboratory data. Scottish centres were excluded from blood pressure, calcium, cholesterol and PTH analyses as corresponding data were not provided.

\section{Results and conclusions}

Table 3.12 shows that $13.4 \%$ of the prevalent transplant population (3,603 patients), had moderate to advanced renal impairment of eGFR $<30 \mathrm{ml} / \mathrm{min} /$
$1.73 \mathrm{~m}^{2}$. The table also demonstrates that patients with failing grafts achieved UK Renal Association standards for some key biochemical and clinical outcome variables less often than dialysis patients. This substantial group of patients represents a considerable challenge, as resources need to be channelled to improve key outcome variables and achieve a safe and timely modality switch to another form of renal replacement therapy.

\section{eGFR slope analysis}

\section{Introduction}

The gradient of deterioration in eGFR (slope) may predict patients likely to have early graft failure. The 
Table 3.13. Differences in median eGFR slope between subgroups of prevalent transplant patients

\begin{tabular}{|c|c|c|c|c|c|c|}
\hline Patient characteristic & & $N$ & $\begin{array}{l}\text { Median } \\
\text { slope }\end{array}$ & $\begin{array}{l}\text { Lower } \\
\text { quartile }\end{array}$ & $\begin{array}{l}\text { Upper } \\
\text { quartile }\end{array}$ & $p$-value \\
\hline Age at transplant & $\begin{array}{c}<40 \\
40-55 \\
>55\end{array}$ & $\begin{array}{l}4,438 \\
5,556 \\
4,499\end{array}$ & $\begin{array}{l}-1.08 \\
-0.36 \\
-0.39\end{array}$ & $\begin{array}{l}-4.15 \\
-2.74 \\
-2.74\end{array}$ & $\begin{array}{l}11.1 \\
16.0 \\
15.8\end{array}$ & $<0.0001$ \\
\hline Ethnicity & $\begin{array}{l}\text { Asian } \\
\text { Black } \\
\text { Other } \\
\text { White }\end{array}$ & $\begin{array}{r}1,313 \\
856 \\
295 \\
11,204\end{array}$ & $\begin{array}{l}-0.93 \\
-1.29 \\
-1.03 \\
-0.52\end{array}$ & $\begin{array}{l}-3.88 \\
-4.27 \\
-4.57 \\
-2.95\end{array}$ & $\begin{array}{l}16.6 \\
13.2 \\
13.3 \\
13.8\end{array}$ & $<0.0001$ \\
\hline Gender & $\begin{array}{c}\text { Male } \\
\text { Female }\end{array}$ & $\begin{array}{l}8,860 \\
5,633\end{array}$ & $\begin{array}{l}-0.36 \\
-0.91\end{array}$ & $\begin{array}{l}-2.78 \\
-3.80\end{array}$ & $\begin{array}{l}15.7 \\
12.5\end{array}$ & $<0.0001$ \\
\hline Diabetes & $\begin{array}{c}\text { Non-diabetic } \\
\text { Diabetic }\end{array}$ & $\begin{array}{r}12,210 \\
2,008\end{array}$ & $\begin{array}{l}-0.47 \\
-1.23\end{array}$ & $\begin{array}{l}-2.95 \\
-4.01\end{array}$ & $\begin{array}{l}15.1 \\
10.6\end{array}$ & $<0.0001$ \\
\hline Year of transplant & $\begin{array}{l}2002 \\
2003 \\
2004 \\
2005 \\
2006 \\
2007 \\
2008 \\
2009 \\
2010 \\
2011\end{array}$ & $\begin{array}{r}787 \\
972 \\
1,138 \\
1,134 \\
1,434 \\
1,572 \\
1,804 \\
1,876 \\
1,943 \\
1,833\end{array}$ & $\begin{array}{l}-0.61 \\
-0.55 \\
-0.32 \\
-0.17 \\
-0.59 \\
-0.66 \\
-0.58 \\
-0.93 \\
-0.80 \\
-0.40\end{array}$ & $\begin{array}{l}-2.32 \\
-2.34 \\
-2.11 \\
-2.07 \\
-2.66 \\
-2.71 \\
-2.96 \\
-3.78 \\
-4.53 \\
-5.93\end{array}$ & $\begin{array}{l}05.8 \\
08.6 \\
10.6 \\
13.0 \\
11.2 \\
11.3 \\
14.3 \\
13.6 \\
24.9 \\
44.2\end{array}$ & $<0.001$ \\
\hline $\begin{array}{l}\text { Status of transplant } \\
\text { at end of follow-up }\end{array}$ & $\begin{array}{c}\text { Died } \\
\text { Failed } \\
\text { Re-transplanted } \\
\text { Functioning }\end{array}$ & $\begin{array}{r}1,006 \\
1,029 \\
54 \\
12,404\end{array}$ & $\begin{array}{l}-0.88 \\
-6.23 \\
-3.83 \\
-0.29\end{array}$ & $\begin{array}{r}-3.98 \\
-11.96 \\
-6.80 \\
-2.51\end{array}$ & $\begin{array}{r}19.4 \\
-2.90 \\
-1.47 \\
16.0\end{array}$ & $<0.001$ \\
\hline
\end{tabular}

eGFR slope and its relationship to specific patient characteristics are presented here.

\section{Methods}

All UK patients aged $\geqslant 18$ years receiving a renal transplant between 1st January 2002 and 31st December 2011, were considered for inclusion. A minimum duration of 18 months graft function was required and three or more creatinine measurements from the second year of graft function onwards were used to plot eGFR slope. If a transplant failed but there were at least three creatinine measurements between one year post-transplant and graft failure, the patient was included but no creatinine measurements after the quarter preceding the recorded date of transplant failure were analysed.

Slopes were calculated using linear regression, assuming linearity, and the effect of age, ethnicity, gender, diabetes, donor type, year of transplant and current transplant status were analysed. $P$ values were calculated using the Kruskal-Wallis test. eGFR was calculated using the CKD-EPI equation and results expressed as $\mathrm{ml} / \mathrm{min} / 1.73 \mathrm{~m}^{2} /$ year. The CKD-EPI equation was used in preference to the MDRD formula as it is thought to have a greater degree of accuracy at higher levels of eGFR [11].

\section{Results and conclusions}

The study cohort consisted of 14,493 patients. The median GFR slope was $-0.58 \mathrm{ml} / \mathrm{min} / 1.73 \mathrm{~m}^{2} /$ year (table 3.13). The gradient was steeper for Black recipients $\left(-1.3 \mathrm{ml} / \mathrm{min} / 1.73 \mathrm{~m}^{2} /\right.$ year$)$, in keeping with previously published data suggesting poorer outcomes for this group $[12,13]$. There was no statistically significant difference in eGFR slope in recipients of deceased donor kidneys $\left(-0.58 \mathrm{ml} / \mathrm{min} / 1.73 \mathrm{~m}^{2} /\right.$ year $)$ compared to patients who received organs from live donors $\left(-0.57 \mathrm{ml} / \mathrm{min} / 1.73 \mathrm{~m}^{2} /\right.$ year). Female patients had a steeper slope $(-0.91 \mathrm{ml} / \mathrm{min} /$ 
$1.73 \mathrm{~m}^{2} /$ year $)$ than males $\left(-0.36 \mathrm{ml} / \mathrm{min} / 1.73 \mathrm{~m}^{2} /\right.$ year $)$, as did diabetic patients $\left(-1.23 \mathrm{ml} / \mathrm{min} / 1.73 \mathrm{~m}^{2} /\right.$ year $)$ compared to non-diabetic patients $\left(-0.47 \mathrm{ml} / \mathrm{min} / 1.73 \mathrm{~m}^{2} /\right.$ year). The slope was steeper in younger recipients, possibly reflecting increased risk of immunological damage. As might be expected, the steepest slope was in patients where the transplant subsequently failed. This analysis has assumed linearity of progression of fall in GFR and further work is underway to characterise the patterns of progression more precisely.

The findings in this study differ slightly from previous UKRR work exploring eGFR changes in transplant recipients [14]. This identified that male donor to female recipient transplantation, younger recipients, diabetes, white ethnicity, and human leukocyte antigen (HLA) mismatch were associated with faster decline in eGFR. These differences may be explained by patients with eGFR $>60 \mathrm{ml} / \mathrm{min} / 1.73 \mathrm{~m}^{2}$ at one year posttransplantation being excluded and the more complex multivariable model used in the previous work. Udayaraj and colleagues [14] also adjusted for factors such as HLA mismatch and donor age, which were not available for the patients studied in this chapter.

\section{Cause of death in transplant recipients}

\section{Introduction}

Differences in causes of death between dialysis and transplant patients may be expected due to selection for transplantation and use of immunosuppression. Chapter 5 includes a more detailed discussion on cause of death in dialysis patients.

\section{Methods}

The cause of death is sent by renal centres as an ERA-EDTA registry code. These have been grouped into the following categories: cardiac disease, cerebrovascular disease, infection, malignancy, treatment withdrawal, other and uncertain.

Some centres have high data returns to the UKRR regarding cause of death, whilst others return no information. Provision of this information is not mandatory. Analysis of prevalent patients included all those aged over 18 years and receiving RRT on 1st January 2013.

\section{Results and conclusions}

Table 3.14 and figure 3.11 show the differences in the cause of death between prevalent dialysis and transplant patients. Table 3.15 shows the cause of death for prevalent

Table 3.14. Cause of death by modality in prevalent RRT patients on $1 / 1 / 2013$, who died in 2013

\begin{tabular}{|c|c|c|c|c|c|c|}
\hline \multirow[b]{2}{*}{ Cause of death } & \multicolumn{2}{|c|}{ All modalities } & \multicolumn{2}{|c|}{ Dialysis } & \multicolumn{2}{|c|}{ Transplant } \\
\hline & $N$ & $\%$ & $N$ & $\%$ & $N$ & $\%$ \\
\hline Cardiac disease & 734 & 23 & 647 & 24 & 87 & 17 \\
\hline Cerebrovascular disease & 136 & 4 & 111 & 4 & 25 & 5 \\
\hline Infection & 664 & 21 & 531 & 20 & 133 & 26 \\
\hline Malignancy & 311 & 10 & 186 & 7 & 125 & 24 \\
\hline Treatment withdrawal & 525 & 16 & 517 & 19 & 8 & 2 \\
\hline Other & 660 & 21 & 543 & 20 & 117 & 23 \\
\hline Uncertain & 186 & 6 & 161 & 6 & 25 & 5 \\
\hline Total & 3,216 & & 2,696 & & 520 & \\
\hline No cause of death data & 1,353 & 30 & 1,130 & 30 & 223 & 30 \\
\hline
\end{tabular}

Table 3.15. Cause of death in prevalent transplant patients on $1 / 1 / 2013$ by age, who died in 2013

\begin{tabular}{|c|c|c|c|c|c|c|}
\hline \multirow[b]{2}{*}{ Cause of death } & \multicolumn{2}{|c|}{ All age groups } & \multicolumn{2}{|c|}{$<65$ years } & \multicolumn{2}{|c|}{$\geqslant 65$ years } \\
\hline & $N$ & $\%$ & $N$ & $\%$ & $N$ & $\%$ \\
\hline Cerebrovascular disease & 25 & 5 & 11 & 4 & 14 & 5 \\
\hline Infection & 133 & 26 & 65 & 25 & 68 & 27 \\
\hline Malignancy & 125 & 24 & 73 & 28 & 52 & 20 \\
\hline Uncertain & 25 & 5 & 9 & 3 & 16 & 6 \\
\hline Total & 520 & & 264 & & 256 & \\
\hline No cause of death data & 223 & 30 & 110 & 29 & 113 & 31 \\
\hline
\end{tabular}




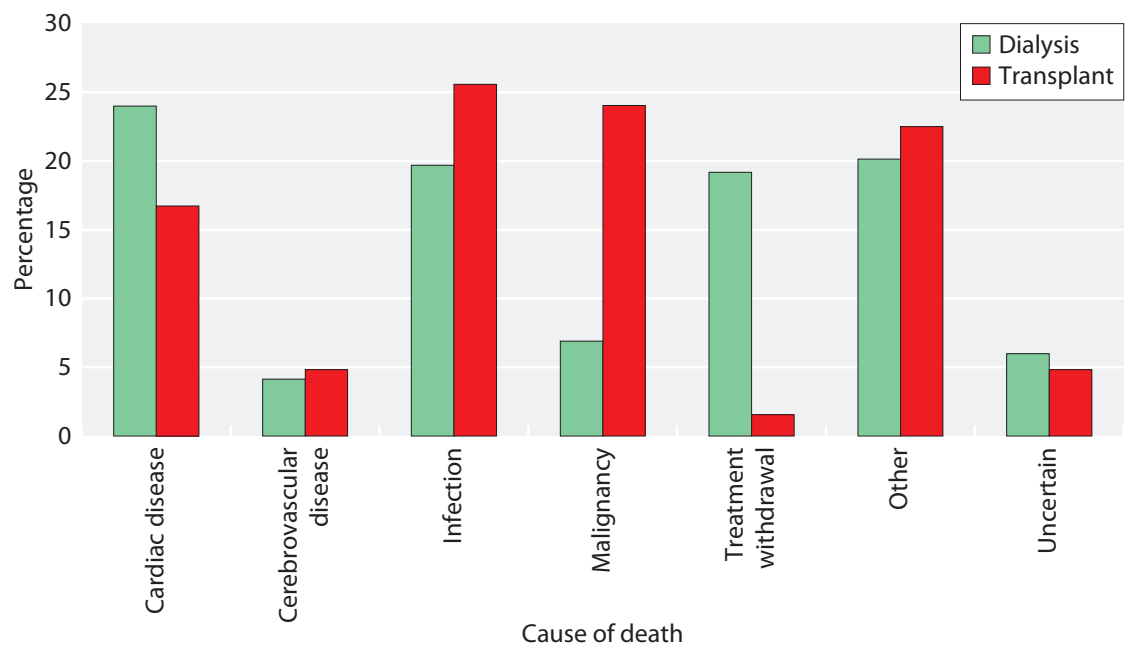

Fig. 3.11. Cause of death by modality for prevalent patients on $1 / 1 / 2013$, who died in 2013 transplant patients by age. Death due to cardiovascular disease was less common in transplanted patients than in dialysis patients, perhaps reflecting the cardiovascular screening undertaken during transplant work-up; transplant recipients are a pre-selected lower risk group of patients. The leading causes of death amongst transplant patients were infection (26\%), malignancy (24\%) and other (23\%). There has been a reduction over time in the proportion of deaths in transplant patients attributed to cardiovascular or stroke disease (43\% in 2003 compared to $22 \%$ in 2013) with an increase in the proportion ascribed to infection or malignancy (30\% in 2003 compared to $50 \%$ in 2013). This change has also been reported in other registries, e.g. ANZDATA (http://www.anzdata.org.au) and may reflect better management of cardiovascular risk (although table 3.12 shows blood pressure management remained suboptimal). Explanations for the rising death rate secondary to malignancy may include the increasing age of transplant recipients and the increased intensity of immunosuppressive regimens leading to complications of over-immunosuppression.

Conflicts of interest: Dr I MacPhee has received research funding and speaker honoraria from Astellas.

\section{References}

1 Ansell D, Tomson CRV: UK Renal Registry 11th Annual Report (December 2008) Chapter 15 The UK Renal Registry, UKRR database, validation and methodology. Nephron Clin Pract 2009;111(suppl 1): c277-c285

2 Pruthi R, Ravanan R, O’Neill J, Roderick P, Pankhurst L, Udayaraj U: 15th annual report: Chapter 9 centre variation in access to renal transplantation in the UK (2006-2008). Nephron Clin Pract. 2013; 123(suppl 1):183-93. doi: 10.1159/000353328

3 Pruthi R, Casula A, MacPhee I: UK Renal Registry 16th annual report: Chapter 3 demographic and biochemistry profile of kidney transplant recipients in the UK in 2012: national and centre-specific analyses. Nephron Clin Pract. 2013;125(1-4):55-80. doi: 10.1159/000360022

-4 Bosma RJ, Doorenbos CRC, Stegeman CA, Homan van der Heide JJ, Navis G: Predictive Performance of Renal Function Equations in Renal Transplant Recipients: An analysis of Patient Factors in Bias. Am J Transplant 2005;5:2183-2203

5 Froissart M, Rossert J, Jacquot C, Paillard M, Houillier P. Predictive Performance of the Modification of Diet in Renal Disease and Cockcroft-Gault Equations for Estimating Renal Function. J Am Soc Nephrol. 2005;16:763-773
6 Hariharan, S, McBride MA, Cherikh WS, Tolleris CB, Bresnahan BA, Johnson CP: Post-transplant renal function in the first year predicts long-term kidney transplant survival. Kidney Int 2002;62:1:311-318

7 UK Renal Association Clinical Practice Guidelines Committee: Anaemia of CKD, 5th Edition. 2010. http://www.renal.org/clinical/ GuidelinesSection/AnaemiaInCKD.aspx

8 UK Renal Association Clinical Practice Guidelines Committee: Guideline 3.7: Target haemoglobin. 2007 RA Guidelines - Complications of CKD, 4th Edition. 2007. http://www.renal.org/Clinical/GuidelinesSection/ ComplicationsofCKD.aspx

9 UK Renal Association Clinical Practice Guidelines Committee: Guideline: Post-operative Care of the Kidney Transplant Recipient, 5th Edition. 2011. http://www.renal.org/Clinical/GuidelinesSection/ Post-operative-Care-Kidney-Transplant-Recipient.aspx

10 UK Renal Association Clinical Practice Guidelines Committee: Guideline 2.1: Treatment of patients with CKD. 2007 RA Guidelines - CKD, 4th Edition. 2007. http://www.renal.org/Clinical/GuidelinesSection/ CKD.aspx

11 White CA, Akbari A, Doucette S, Fergusson D, Knoll GA: Estimating Glomerular Filtration Rate in Kidney Transplantation: Is the New 
Chronic Kidney Disease Epidemiology Collaboration Equation Any Better? Clin Chem 2010;56:3:474-477

$\checkmark 12$ Ng FL, Holt DW, Chang RWS, MacPhee IAM: Black renal transplant recipients have poorer long-term graft survival than CYP3A5 expressers from other ethnic groups. Nephrol Dial Transplant 2010;25:628634
13 Isaacs RB, Nock SL, Spencer CE, Connors AF Jr, Wang XQ, Sawyer R, Lobo PI: Racial disparities in renal transplant outcomes. Am J Kidney Dis 1999;34:4:706-712

14 Udayaraj U, Casula A, Ansell D, Dudley CRK, Ravanan R: Chronic Kidney Disease in Transplant Recipients - Is It Different From Chronic Native Kidney Disease? Transplantation 2010;90:7:765-770 


\section{Appendix 1: Reporting status of audit measures}

Table 3.16. Showing the reporting status of the recommended Renal Association Audit Measures for the Post-operative Care of Kidney Transplant Recipients in the 17th Annual Report

\begin{tabular}{ccc}
\hline & Included in \\
RA audit measure & UKRR annual \\
report? & Reason for non-inclusion \\
\hline
\end{tabular}

1. Proportion of blood results available for review, and reviewed, within 24 hours

2. Proportion of units with a written follow-up schedule available to all staff and patients

3. Percentage of patients accessing their results through Renal Patient View

4. Percentage of total patients assessed in an annual review clinic

5. Percentage of total patients receiving induction with ILRAs and TDAs

6. Percentage of de novo KTRs receiving tacrolimus

7. Percentage of de novo KTRs receiving MPA based immunosuppression

8. Percentage of de novo KTRs receiving corticosteroid maintenance therapy

9. Use of generic agents

10. Severity of biopsy proven acute rejection (BPAR) recorded by BANFF criteria

11. Percentage of KTRs with BPAR in first 3 months and first 12 months

12. Percentage of KTRs requiring TDAs to treat rejection in first year

13. Complication rates after renal transplant biopsy

14. Proportion of patients receiving a target blood pressure of 130/ $80 \mathrm{mmHg}$ or $125 / 75 \mathrm{mmHg}$ in the presence of proteinuria $($ PCR $>100$ or ACR $>70)$

15. Proportion of patients receiving an ACE inhibitor or angiotensin receptor blocker

16. Proportion of patients with proteinuria assessed by dipstick and, if present, quantified at each clinic visit

17. Proportion of renal transplant recipients with an annual fasting lipid profile

18. Proportion of KTR taking statins (including the type of statin) for primary and secondary prevention of premature cardiovascular disease

19. Proportion of patients on other lipid lowering agents

20. Proportion of patients achieving dyslipidaemia targets

21. Incidence of new onset diabetes after transplantation (NODAT) at three months and at annual intervals thereafter

22. Proportion of patients who require insulin, and in whom remedial action is undertaken - minimisation of steroids and switching of CNIs

23. Proportion of patients with ischaemic heart disease

24. Proportion of patients suffering myocardial infarction

25. Proportion of patients undergoing primary revascularisation
No

No

No

No

No

No

No

No

No

No

No

No

No

No

No

No

No

No

No

Yes

No

No

No

No

No
UKRR does not currently collect these data

UKRR does not currently collect these data

Requires linkage with RPV

UKRR does not currently collect these data

Poor data completeness

Poor data completeness

Poor data completeness

Poor data completeness

UKRR does not currently collect these data UKRR does not currently collect these data

UKRR does not currently collect these data

UKRR does not currently collect these data

UKRR does not currently collect these data

Poor data completeness

Poor data completeness

UKRR does not currently collect these data

UKRR does not currently collect these data

UKRR does not currently collect these data

Poor data completeness

UKRR does not currently collect these data

UKRR does not currently collect these data

Poor data completeness

Poor data completeness

Poor data completeness 
Table 3.16. Continued

Included in

UKRR annual

RA audit measure

26. Proportion of patients receiving secondary prevention with a statin, anti-platelet agents and RAS blockers

27. Proportion of patients who are obese

28. Proportion of patients having screening procedures for neoplasia at the annual review clinic

29. Incidence of CMV disease

30. Rate of EBV infection and PTLD

31. Completeness of records for EBV donor and recipient serology

32. Rates of primary VZV and shingles infection

33. Completeness of records for VZV recipient serology

34. Rates and outcomes of HSV infection

35. Rates of BK viral infection in screening tests

36. Rates and outcomes of BK nephropathy

37. Frequency of bisphosponate use

38. Incidence of fractures

39. Incidence of hyperparathyroidism

40. Incidence of parathyroidectomy

41. Use of cinacalcet

42. Frequency of hyperuricaemia and gout

43. Prevalence of anaemia

44. Prevalence of polycythaemia

45. Pregnancy rates and outcomes

46. Prevalence of sexual dysfunction report?

Reason for non-inclusion

No UKRR does not currently collect these data

No Poor data completeness

No UKRR does not currently collect these data

No Poor data completeness

No UKRR does not currently collect these data

No UKRR does not currently collect these data

No UKRR does not currently collect these data

No UKRR does not currently collect these data

No UKRR does not currently collect these data

No UKRR does not currently collect these data

No UKRR does not currently collect these data

No UKRR does not currently collect these data

No UKRR does not currently collect these data

No Poor data completeness

No UKRR does not currently collect these data

No Poor data completeness

No UKRR does not currently collect these data

Yes

No Poor data completeness

No UKRR does not currently collect these data

No UKRR does not currently collect these data 